\title{
LAVORARE AI TEMPI DELL'ECONOMIA DIGITALE. LE TRASFORMAZIONI DEL LAVORO NEL CONTESTO DEL CAPITALISMO COGNITIVO E DELL'ECONOMIA DELLE PIATTAFORME
}

\author{
LABOR IN THE TIME OF DIGITAL ECONOMY. LABOR TRANS- \\ FORMATIONS IN THE CONTEXT OF COGNITIVE CAPITALISM \\ AND THE PLATFORM ECONOMY
}

\author{
Luca Raffini \\ Università di Genova (Italia) \\ luca.raffini@edu.unige.it
}

\begin{abstract}
Astratto
Il contributo ha come oggetto le trasformazioni del lavoro nel contesto del capitalismo cognitivo e dell'economia delle piattaforme. Si evidenziano rischi e opportunità connessi a questa trasformazione e se ne indaga criticamente il "lato oscuro". I giovani sono socializzati a una nuova etica del lavoro. Vivono una esperienza di lavoro individualizzata, frammentata e destandardizzata. Esperiscono il superamento dei confini tra tempo di lavoro e tempo di non lavoro, così come dei confini tradizionalmente posti tra lavoro, formazione, impegno sociale e tempo libero. Le nuove forme organizzative e la nuova etica del lavoro trovano espressione in una pluralità di pratiche e di nuovi ambiti lavorativi, dando espressione a pratiche innovative ma anche generando il rischio di nuove forme di sfruttamento e di alienazione. Le prime sono solitamente associate allo sviluppo di pratiche come l'economia collaborativa e l'impresa sociale, mentre i rischi accompagnano lo sviluppo della cosiddetta GIG Economy.
\end{abstract}

\section{Parole chiave}

Capitalismo cognitivo, Trasformazioni del lavoro, Economia digitale, Economia delle piattaforme 


\begin{abstract}
The contribution concerns the transformations of work in the context of cognitive capitalism and the platform economy. The risks and opportunities associated with this transformation are highlighted and the "dark side" is critically investigated. Young people are socialized to a new work ethic. They live an individualized, fragmented and de-standardized work experience. They experience overcoming the boundaries between work time and non-work time, as well as the boundaries traditionally placed between work, training, social engagement and leisure. The new organizational forms and the new work ethic find expression in a plurality of practices and new working environments, giving expression to innovative practices but also generating the risk of new forms of exploitation and alienation. The former are usually associated with the development of practices such as collaborative economics and social enterprise, while risks accompany the development of the so-called GIG Economy.
\end{abstract}

\title{
Keywords
}

Cognitive capitalism, Transformations of work, Digital economy, Platform economy

\section{Extended abstract}

The contribution concerns the transformations of work in the context of cognitive capitalism and the platform economy. The central question that we try to answer with the article is how, and with what social, economic and political impact, digital capitalism - developed as a consequence of the fourth industrial revolution - transforms work. The risks and opportunities associated with this transformation are highlighted and the "dark side" is critically investigated, arguing that rather than an "end of work", there is an end to wage labor accompanied by the rights and protections won by workers in "thirty glorious years". Working hours increase, while protections, rights and remuneration decrease. The article reconstructs, first of all, the key elements of change that contribute to transforming the experience of work, with particular reference to the new generations, paying attention to both technological change and social change in general, and investigating the link between two. It defines the new scenarios of capitalism and analyzes how these contribute to transforming work, on an organizational, value and identity level. Young people, in a context of individualization, fragmentation and de-standardization of work, have fully internalized an approach oriented towards adaptability and the pursuit of employability, such as new pivotal principles that guide the relationship with work. This differs, at times and in the workplace, to become confused within everyday life, generating progressive indistinction between life time and work time. This form of overlapping favors practices of (self) exploitation and the affirmation of new forms of subsumption, that is, of putting value on the 
relationships and products of human action, even outside the working environment. Precariousness tends to assume an existential, as well as a working, dimension, and extends to the family, social and political sphere, as well as economic and permeates the relationship between work, citizenship and social identity. Less and less work is part of a collective narrative, to become a private matter. The fragmentation and volatility of the work lead to the overcoming of the underlying element on which the identification - and therefore the collective action - was based in industrial capitalism: the sharing of a large number of workers of the same labor contract, of the same tasks, the same hours, the same conditions. If these are the basic and transversal elements that define the transformations of the work, in the second part of the essay the main areas and the main expressions assumed by the transformation of the work are investigated in depth. By paying attention to the different, clear and dark faces of change, attention is paid to the practices of the sharing economy and the economy of collaboration and to the development of social enterprise and civil economy, as a meeting place for action, economic and social action, but also how the economy of platforms, and in particular the so-called GIG Economy, can create new forms of deregulation and exploitation. The work produced in the context of the GIG Economy, in fact, very little conforms to the rhetoric of cognitive works, highly rewarding, promised by the knowledge society. The opposite, rather, constitute a sort of "digital Taylorism" whose workers, at the margin of the welfare system; in some respects assume typical features of the mass worker of Fordism, and for other characteristics typical of the pre-Fordist phase, in terms of exploitation, alienation, and commodification. For many young people, the new work promised by the economy from digital capitalism and the platform economy has this face today.

\section{INTRODUZIONE. MEDIA DIGITALI E MUTAMENTO SOCIALE}

Il mutamento tecnologico ha una parte importante nella trasformazione della società, ma non può essere assunto come unica, e neanche principale, variabile esplicativa. Qualsiasi riflessione in merito al rapporto tra mutamento tecnologico e mutamento sociale deve piuttosto interrogarsi sui loro rapporti di codeterminazione. Ciò vale a riguardo delle trasformazioni delle relazioni sociali, dei processi di socializzazione, dei fenomeni politici, così come delle relazioni economiche e del lavoro, su cui si concentra il presente contributo.

Da sempre le innovazioni tecnologiche sono state accompagnate da profonde trasformazioni sociali. Hanno generato rischi e opportunità e alimentato speranze e paure. È successo con la prima rivoluzione industriale - fondata sull'introduzione della macchina a vapore -, con la seconda rivoluzione industriale - coincidente con l'utilizzo dell'elettricità -, con la terza rivoluzione industriale - caratterizzata dall'automazione e dalla digitalizzazione. Non sfugge 
a questa tensione l'attuale "quarta rivoluzione industriale", al cui centro vi sono le nuove opportunità rese possibili dall'utilizzo delle piattaforme e dal ricorso all'intelligenza artificiale, in un contesto di crescente sovrapposizione $\mathrm{e}$ ibridazione tra ambiente fisico, digitale e biologico (Schwab 2018).

In ognuno di questi passaggi storici si sono confrontati entusiasti - convinti che l'innovazione tecnologica avrebbe automaticamente contribuito a migliorare le condizioni di vita dell'uomo, liberandolo dal lavoro e aumentando le risorse a disposizione di ognuno, e scettici, convinti che la tecnologie avrebbero, al contrario, sostituito il lavoro degli uomini, aumentando la povertà e rendendo possibili nuove forme di sfruttamento, alienazione e mercificazione. Le due visioni, a ben vedere, rappresentano due facce della stessa medaglia, e il prevalere di una o dell'altra non dipende da alcun automatismo, ma dalla capacità delle società di guidare il cambiamento, generando nuovi equilibri e sviluppando nuove forme di regolazione. Poiché il mutamento tecnologico precede di norma il mutamento organizzativo, ed entrambi sono assai più veloci rispetto al mutamento istituzionale, è normale che le fasi di grande trasformazione siano accompagnate da una percezione di crisi. Questa tende oggi a essere un carattere strutturale, più che contingente e transitorio, a fronte dell'accelerato e continuo mutamento, che genera incertezza e insicurezza. D'altra parte, già a partire dalla prima rivoluzione industriale, la "grande trasformazione" descritta da Polany (2000), ha evidenziato che progresso tecnologico e crescita economica non coincidono necessariamente con il miglioramento delle condizioni di vita della maggioranza della popolazione e che, quindi, non vi è alcun automatismo tra sviluppo tecnologico, sviluppo economico e progresso sociale (O'Reilly et al. 2018). Già Engels, nella sua prima, straordinaria, indagine sulle condizioni di vita del nuovo proletariato, aveva evidenziato come l'avvento della società industriale avesse generato sfruttamento, anomia, deprivazione e marginalità sociale, oltre che alienazione. La "quadratura del cerchio" tra sviluppo economico, consolidamento della democrazia, benessere e giustizia sociale, a cui le società avanzate sono approdate nei decenni successivi, e in particolare nei "gloriosi trent'anni" successivi al secondo conflitto mondiale non è avvenuta in virtù dell'intervento di una "mano invisibile" ma in conseguenza di un intervento regolativo e redistributivo da parte dello Stato, ottenuto a seguito di conflitti e rivendicazioni. Ciò che oggi appare, se non assente, altamente indebolita, è proprio la capacità regolativa della politica sull'economia, e quindi sul lavoro.

Va, non di meno, considerato che le spinte impresse dalla quarta rivoluzione industriale sul lavoro si inseriscono in un quadro trasformativo pregresso, rispetto al quale, è la nostra ipotesi, non introducono una discontinuità. Piuttosto, amplificano, al contempo aumentandone la complessità, le dinamiche e gli orientamenti preesistenti, che convergono nel trasformare in profondità tanto 
l'organizzazione e la regolazione del lavoro, quanto il rapporto tra lavoro, identità, cittadinanza e, infine, i valori che compongono l'etica del lavoro.

A partire da questo presupposto, l'interrogativo centrale a cui si cerca di rispondere con l'articolo è in che modo, e con quale impatto sociale, economico e politico, il capitalismo digitale - sviluppatosi come conseguenza della quarta rivoluzione industriale, trasforma il lavoro. L'ipotesi è che le nuove generazioni siano oggi sospese tra un modello lavorativo che non esiste più, se non in forma residuale, quello del lavoro salariato, di tipo standard, e un nuovo modello che, ad oggi, pone più interrogativi che certezze, e di cui si intravedono in modo assai più chiaro i rischi che le opportunità, anche in virtù del ritardo con cui il mutamento dell'esperienza lavorativa è seguito da un mutamento delle forme di regolazione e di tutela.

Uno degli effetti è la crescente polarizzazione. Il superamento del modello di lavoro standard, che vede il lavoratore impiegato per tutta la vita nello stesso tipo di occupazione, con contratto salariato, e orari di lavoro rigidamente definiti, solo per una minoranza sembra dischiudere la prospettiva di un rapporto con il lavoro più appagante, sul piano economico, identitario e sociale, fatto di flessibilità, autonomia, dinamismo, innovazione, creatività. Per la maggioranza dei lavoratori le trasformazioni del lavoro appaiono più subite che gestite, e si manifestano in forma di precarietà, incertezza, diminuzione delle tutele, dilatazione dell'orario di lavoro, diminuzione del reddito, in una situazione, per lo più, che vede sempre più i rischi trasferirsi dai datori di lavoro - e dallo Stato - al singolo lavoratore. Lo scenario utopico, in cui il progresso tecnologico, l'aumento della produttività, e il progressivo shift verso il settore dei servizi e della conoscenza, avrebbe "liberato" gli uomini dal lavoro, nel senso che avrebbe reso possibile una diminuzione complessiva del tempo lavorativo, nel quadro di un miglioramento delle condizioni di vita di e redistribuzione della ricchezza, si è rovesciato in uno scenario distopico. Più che a una "fine del lavoro", si assiste a una fine del lavoro salariato e accompagnato dai diritti e dalle tutele conquistate dai lavoratori nei "trenta anni gloriosi". Le ore di lavoro e, nel complesso, la parte di vita messa a valore, aumentano, mentre diminuiscono tutele, diritti, retribuzione. Ciò perché si estende, fino a coprire potenzialmente ogni ambito di vita, l'ambito in cui produciamo valore, in forma diretta o indiretta (producendo contenuti, ampliando le proprie reti e le proprie competenze, stabilendo connessioni, tramite il coinvolgimento in attività che arricchiscono il proprio $\mathrm{cv}$ ), ma il valore prodotto è per lo più messo a profitto da altri. Sembra oggi di assistere a una radicalizzazione delle dinamiche - e dei rischi e delle opportunità a queste connesse - iniziate a profilarsi negli anni settanta del secolo scorso, allorché si coglievano i segnali di una trasformazione radicale del lavoro a seguito del passaggio ad una società postindustriale e postfordista, fondata sull'informazione, i servizi e la conoscenza. In seguito, con i concetti di capitalismo molecolare e cognitivo, si è sancito la fuoriuscita del lavoro dalla 
fabbrica e l'avvento dell'operaio sociale. Assistiamo alla crisi delle forme tradizionali di rappresentanza sindacale, ritagliate sul lavoratore salariato, inquadrato con contratti standard, che presta il proprio lavoro in luoghi, orari, modalità predefinite, ripetute, del tutto identiche ai propri colleghi di lavoro. Da questo momento, il lavoro "atipico" si diffonderà in modo esponenziale, fino a comprendere la grande maggioranza degli inquadramenti contrattuali dei giovani, e non solo. Il lavoro atipico si diversifica, negli orari e nei luoghi di lavoro, e, soprattutto nel caso del lavoro di tipo cognitivo, si frammenta all'interno della vita quotidiana. Se prima si lavorava per lo più nel proprio ufficio, in orari ben definiti, ora si lavora a casa, connessi con il proprio laptop in treno, in aeroporto, al tavolo di un caffè. Come fanno sempre più spesso i "nomadi digitali". E lo si fa, sempre più, in una condizione di solitudine. Perché si lavora da casa, perché il lavoro si risolve per lo più in un rapporto solipsistico con un computer, un telefono, con App (ci riferiamo ai lavoratori che effettuano consegne) o altre interfacce elettroniche, come nei centri logistici di Amazon, al di fuori di qualsiasi forma di socialità. O perché, pur condividendo lo stesso tipo di lavoro, nello stesso ambiente, ed avendo rapporti di interscambio, si hanno diversi rapporti contrattuali, o si lavoro per imprese diverse. Precarietà, frammentazione, individualizzazione del rapporto di lavoro, comportano un chiaro riflesso sulle capacità di sviluppare identificazioni comuni e di produrre mobilitazioni collettive, in un contesto in cui le condizioni di lavoro appaiono vieppiù peggiorare. Come si conciliano queste tendenze con le retoriche che individuano nel capitalismo digitale il luogo ideale di generazione di innovazione sociale, di inedite forme di incontro tra azione economica e sociale, dell'affermazione di rapporti di lavoro appaganti, autonomi, flessibili, fondati sulla relazionalità? A ben vedere, parte dell'ambiguità che caratterizza l'affermazione delle nuove modalità di organizzazione del lavoro negli ambienti digitali è insita nelle dinamiche da questi attivate. Ma è in larga parte frutto di una indeterminatezza definitoria e di una retorica dell'innovazione che tende ad accomunare pratiche, esperienze, modelli produttivi e di lavoro, assai diversi tra loro, e accomunati solo dall'utilizzo delle nuove tecnologie. Si tende, per esempio, a fare coincidere, come se fossero la stessa cosa, capitalismo digitale ed economia della condivisione o della collaborazione, estendendo per questa via a ogni forma di capitalismo digitali i supposti effetti benefici delle seconde. In realtà, molte delle esperienze e delle pratiche comunemente associate all'economia della condivisione e della collaborazione assumono solo marginalmente i tratti distintivi di queste e, al contrario, rivelano impatti potenzialmente assai negativi in termini di trasformazioni del lavoro. Si rivelano non forme di economia alternativa ma nuove - e più efficaci - modalità di realizzazione di forme di capitalismo estrattive, che dietro la retorica dell'innovazione celano, di fatto, approcci orientati alla deregolamentazione e tendenti allo sfruttamento. 
Per fare chiarezza sulla diversità di fenomeni che sono impropriamente oggi raccolti sotto categorie ombrello assai indeterminate e vaghe è quindi necessario, in primo luogo, fare chiarezza sull'estrema varietà delle forme concrete con cui questi si presentano, indagandone luci e ombre e analizzandone gli impatti sulle condizioni di lavoro. Le luci, in prima approssimazione, possono essere fatte coincidere con le pratiche di economia della condivisione e della collaborazione, in cui le tecnologie digitali si pongono al servizio di inedite forme di incontro tra economico e sociale. Le ombre si profilano allorché, nell'economia delle piattaforme, l'impiego delle tecnologie si pone a fondamento di pratiche di estrema parcellizzazione e mercificazione del lavoro.

Il contributo è strutturato nel seguente modo. Nel secondo paragrafo si inquadrano gli elementi chiave di mutamento che contribuiscono a trasformare l'esperienza del lavoro, con particolare riferimento alle nuove generazioni. Partendo dal presupposto che i confini tra le due espressioni assunte dal nuovo modello economico sono estremamente labili e porosi, dando forma a un'estrema ambivalenza, si indagano, quindi, nel terzo paragrafo, le dinamiche "civilizzatrici" dell'economia. Si analizza il contributo impresso dalle tecnologie al perseguimento di modelli economici, sociali ed ambientali orientati alla sostenibilità; le prospettive di sviluppo di un'innovazione nel modo in cui pensiamo, regoliamo e viviamo il lavoro, nel modo in cui pensiamo il rapporto tra tempo di lavoro e tempo di non lavoro, in cui definiamo le relazioni tra produzione e consumo, e, soprattutto, nel modo in cui ripensiamo il rapporto tra attività lavorative, retribuzione e diritti sociali. Si guarda, alle prospettive di sviluppo di nuove forme di imprenditorialità, che coniugano competitività e collaborazione, profitto e orientamento pro-sociale, suggerendo una trasformazione virtuosa dell'esperienza del lavoro, e la possibilità di un riconoscimento della natura plurale del lavoro, oltre i confini del lavoro salariato di tipo standard con cui è stato assimilato il lavoro nella società novecentesca. Il quarto paragrafo è dedicato all'affermazione di nuovi modi di fare impresa, che trovano espressione nell'affermazione dell'impresa sociale, dell'impresa $4.0 \mathrm{e}$ nella prospettiva del societing. Nel quinto paragrafo l'attenzione si sposta sul vasto ambito di esperienze di capitalismo digitale in cui l'utilizzo delle piattaforme, e anche delle forme di relazionalità e di cooperazione orizzontale eventualmente presenti, sono messe al servizio del profitto privato. Lo sguardo, ora, si concentra sui processi di deregolamentazione, precarizzazione e frammentazione del lavoro: dinamiche pregresse che rischiano di trovare nelle pratiche economiche fondate sull'utilizzo delle piattaforme digitali un fertile ambito di sviluppo, favorendo l'incremento del lavoro sottoretribuito o financo del lavoro gratuito. Nel paragrafo conclusivo, infine, si propone una riflessione critica sui fenomeni trasformativi in atto e sulle sfide che li accompagnano, anche sul piano politico. 


\section{CINQUANTA SFUMATURE DI LAVORO. DAL CAPITALISMO INDUSTRIALE AL CAPITALISMO DIGITALE}

Il lavoro, tradizionalmente elemento di congiunzione tra l'individuo e la società, capace di strutturare l'identità culturale, sociale e politica, diventa sempre più un fatto privato, più che un'esperienza collettiva. $\mathrm{Al}$ suo centro si pongono vieppiù i principi dell'adattamento, dell'attivazione, dell'assunzione individuale delle responsabilità dei propri successi quanto delle colpe di eventuali fallimenti. Per comprendere la nuova etica del lavoro nelle giovani generazioni dobbiamo partire da lontano, dal Movimento del 1968, di cui si celebra quest'anno il cinquantesimo anniversario. Proprio il 68 è associato allo sviluppo di un nuovo orientamento valoriale, fondato sul rifiuto della gerarchia, della verticalità, e che enfatizza, invece, la libertà, la creatività, la libera espressione di sé. Si tratta della costellazione valoriale che Inglehart ha efficacemente sintetizzato con il concetto di valori postmaterialisti (1977), tipici delle generazioni socializzate in un contesto che, a differenza di quella dei loro genitori, garantiva sicurezza materiale e benessere. A sostenere i valori posmaterialisti sono i Baby-boomers, che si oppongono alla generazione dei loro genitori, cresciuti nel contesto di deprivazione e di sofferenza della guerra, e che invece pongono al centro delle loro aspirazioni valori prettamente materialisti, sintetizzati nelle tre M: mestiere, moglie/marito e macchina. I valori postmaterialisti si consolidano nelle generazioni successive, "senza padri e senza maestri" (Ricolfi, Sciolla 1980), che compiono un progressivo distacco dalla politica, almeno nelle sue forme istituzionali. Questo trend sembra proseguire inesorabile fino agli anni novanta, in quelle che sono state definite la generazione invisibile e la generazione $\mathrm{X}$.

Boltanski e Chiapello (2014) offrono una lettura più articolata e complessa dell'affermazione dei valori postmaterialisti, che ne rivela le connessioni con le trasformazioni del capitalismo. I valori materialisti, ci dicono gli autori, ci parlano del mondo dei partiti di massa e delle ideologie, dello Stato-nazione, ma anche del mondo dell'industrializzazione di massa, la cui figura dominante è l'operaio. I nuovi valori che si affermano a partire dal Sessantotto, a ben vedere, non sono solo i valori che accompagnano l'eclissi della politica e il ritiro nel privato, ma anche $\mathrm{i}$ valori che si pongono a fondamento di una radicale trasformazione del lavoro, in quella che è stata inizialmente definita società postindustriale, poi società dell'informazione, e che ha quindi condotto a nuove declinazioni del capitalismo, variamente definito come "capitalismo molecolare" "cognitivo", "digitale", ecc.

L'enfasi è posta sull'autonomia, sulla flessibilità, sulla creatività, anche a scapito della sicurezza dell'impiego. Il "nuovo spirito del capitalismo", fondato su questi valori, nasce infatti come reazione di fronte a un modello che aveva garantito stabilità e sicurezza al prezzo della libertà e dell'autonomia. La crescente enfasi 
posta su flessibilità e autonomia, a scapito di stabilità e sicurezza, assume connotati diversi in un contesto di peggioramento delle condizioni e delle prospettive lavorative. Allorché non si configura più come una scelta ma come un obbligo, la flessibilità rischia di diventare precarietà, e l'autonomia isolamento e solitudine. I suddetti valori, orientati all'emancipazione degli individui rispetto al controllo degli apparati e delle burocrazie, rischiano oggi di porsi a servizio di un modello economico - e sociale - che si appropria di questi valori per produrre profitto, trasformandoli, paradossalmente, in nuovi, raffinati, strumenti di autosfruttamento e di sussunzione del valore, in un contesto di deregolamentazione e di mercificazione delle relazioni sociali. "La celebrata autonomia e imprenditorialità degli individui è pagata con la distruzione della sicurezza materiale e psicologica. Il nuovo spirito del capitalismo trionfa perché ingloba un bisogno diffuso di autenticità, trasformando in merci da consumare il contenuto delle contestazioni al sistema manifestate dal Sessantotto a oggi. Una forma subdola di sfruttamento che rende impotente o complice la critica sociale condotta finora" (Boltanski, Chiapello 2014). Il nuovo spirito del capitalismo, in sintesi, fa riferimento ai valori espressi dal Sessantotto per porli a servizio di un modello di profitto pervasivo, deregolamentato, che molto ha a che fare con l'affermazione dell'ideologia neoliberista nel frattempo diffusasi a scala globale. Il neoliberismo, a differenza del liberismo tradizionale, celebra il mercato come principio da applicare in ogni ambito della vita sociale, chiedendo allo Stato non di limitare il suo intervento al minimo indispensabile, ma, al contrario, di intervenire attivamente per affermare e imporre i principi e le logiche del mercato in ogni sfera della società (Brenner 2004; Le Gales 2015), per ampliare i margini di autonomia del singolo e ridurre ogni intervento dello Stato di tipo redistributivo. Il nuovo spirito del capitalismo si pone a sostegno dell'antropologia neoliberista - promuovendo la costruzione di un nuovo self neoliberista, dal momento che le virtù dell'autonomia, della flessibilità, della mobilità, della reticolarità sono celebrate quale strumento di competizione individuale, laddove questi principi diventano strumento a supporto dell'attivazione e della performatività (Chicchi, Simone 2015) di individui imprenditori di se stessi. L'individuo è posto, sempre più in condizione di solitudine, a gestire in modo diretto il rapporto con le relazioni di potere, che plasmano le sue relazioni e le sue strategie, facendosi carico - a livello di biografia individuale - delle contraddizioni sistemiche (Beck 1999).

Il nuovo modello di capitalismo, sempre più fondato su deregolamentazione, finanziarizzazione, riduzione delle tutele dei lavoratori, e ritenuto responsabile di un generalizzato aumento di precarietà, vulnerabilità, nuova povertà, "disafiliazione" (Castel 2015), entra in crisi nel primo decennio del nuovo millennio: una crisi che ne svela l'insostenibilità e che alimenta la delegittimazione delle istituzioni ritenute responsabili del suo sviluppo, a partire 
dalle istituzioni europee, in Europa, e dei partiti tradizionali, e che si riflette nel consenso verso partiti e movimenti "anti-establishment", a ogni livello.

Le nuove generazioni - intendendosi con essi i cosiddetti "millenials", nati a partire dagli anni novanta, sono socializzati in un contesto in cui il complesso intreccio tra i fattori sopra analizzati è ormai in atto da molti anni, saldandosi nella percezione che stiamo vivendo un cambio di paradigma, i cui contorni sono però tutt'altro che chiari. Privi dei punti di riferimento che avevano accompagnato le generazioni precedenti plasmandone rappresentazioni, percezioni e valori, vivono pienamente le ambiguità e le ambivalenze delle relazioni economiche e produttive emergenti.

Hanno vissuto in pieno gli effetti della flessibilizzazione e della frammentazione del lavoro, e quindi della crisi economica dell'ultimo decennio, che, oltre ad amplificare i trend preesistenti, ha comportato, soprattutto nei paesi del sud, un incremento di disoccupati e NEET. Il lavoro, quando c'è, per i giovani è vieppiù sotto-retribuito, sotto-qualificato, sotto-tutelato. La consapevolezza di vivere, per la prima volta in decenni, una prospettiva non di miglioramento, ma di peggioramento delle condizioni e delle prospettive, rispetto ai propri genitori, e l'assenza di fiducia nel futuro, spinge i giovani ad adottare un atteggiamento pragmatico, non di rado accompagnato da una precoce disillusione, che si riflette molto nei loro orientamenti valorizzati e nella loro predisposizione all'azione collettiva.

Recenti ricerche concorrono nell'indicare che i giovani, in Italia, manifestano aspettative molto basse in merito al lavoro che svolgeranno nella propria vita, al punto di esprimere, in modo diffuso, la disponibilità a derogare, ovvero accettare livelli inferiori rispetto a quelli che riterrebbero congrui con le loro aspirazioni e aspettative. In particolare, sono vieppiù disposti ad accettare lavori sottoqualificati, sottoretribuiti, sottotutelati, oltre che a incrementare le ore di lavoro, a lavorare nei giorni festivi, con orari spezzati, e completamente modellati sulle esigenze del datore di lavoro. In questo tipo di atteggiamento, è facile individuare un'inversione di tendenza nel processo che - in un contesto di crescente sicurezza economica - ha portato all'affermazione dei valori postmaterialisti (Inglehart 1977), ovvero alla celebrazione della centralità della dimensione dell'affermazione di sé e della qualità delle relazioni. Allo stesso tempo sono pienamente socializzati ai valori caratterizzanti il nuovo spirito del capitalismo, anche al di fuori degli ideali utopici ed emancipativi che, seppur ambiguamente, li caratterizzavano: l'idea che l'individuo è artefice del proprio destino, e che il suo percorso professionale e di vita sia determinato dalle sue scelte, dalla sua capacità di attivazione e dalla sua capacità di essere desiderabile ed impiegabile, sono ampiamente radicate, pur nella consapevolezza che vi siano delle determinanti sistemiche di fondo che incidono su questi processi. 
Provando a sintetizzare, possiamo affermare che i giovani hanno pienamente interiorizzato un approccio che individua nell'adattabilità e nell'impiegabilità (Cuzzocrea 2015) il principio di fondo che orienta i loro comportamenti e le loro strategie. Questi concetti si pongono, in modo alquanto ambiguo e ambivalente, a fondamento delle politiche di attivazione implementate a livello nazionale ed europee, miranti cioè non a garantire lavoro o reddito ai cittadini, ma a offrire loro gli strumenti per essere più impiegabili e che, in modo ancor più ambiguo e ambivalente, trovano espressione nel concetto di resilienza. Si tratta di un concetto mutuato dalle scienze naturali, e che, sul piano individuale e collettivo, significa adattarsi e sopravvivere in una società che è irrimediabilmente segnata da complessità, contingenza e insicurezza (Joseph 2013). Il discorso sulla resilienza pone l'enfasi sulla dimensione della responsabilizzazione, della riflessività e dell'attivazione individuale, estendendo questa logica anche a un'azione collettiva e solidale che sempre più viene privata della sua dimensione conflittuale e antagonista. In questo modo, a ben vedere, dietro l'etichetta della resilienza si nasconde una pluralità di pratiche che spaziano dalla sperimentazione di modelli alternativi al neoliberismo alla sua celebrazione, passando per forme intermedie che possiamo definire di mitigazione (Alteri, Cirulli, Raffini 2018). Il concetto di resilienza è fondamentale per comprendere le possibili implicazioni dei nuovi modelli di economia sperimentati grazie al ricorso intensivo alle piattaforme digitali, poiché queste si caratterizzano in maniera forte per una inedita conciliazione tra economico e sociale che è anche "conseguenza della ritirata della protezione dello Stato e dell'abbandono di molte delle sue responsabilità in quanto a diritti e tutele" (Alaminos e Penalva 2018).

I giovani devono adattarsi al nuovo contesto economico e lavorativo, devono sapere (sapersi) innovare, non guardare nostalgicamente al passato ma trovare nell'attuale contesto di trasformazione gli stimoli per sperimentare nuovi percorsi, nuove strade, nuove combinazioni.

Ma in che direzione muove questa dinamica di trasformazione - a cui si è chiamati ad adattarsi? Quali conseguenze ha rispetto alla capacità delle società avanzate, ed in particolare europee, di conciliare virtuosamente sviluppo economico, democrazia, benessere? Polarizzazione sociale, aumento delle diseguaglianze, e quindi dei cittadini posti in condizioni di vulnerabilità, sono un destino ineludibile?

L'interazione tra mutamento strutturale, mutamento valoriale, evoluzione dei modelli di capitalismo, affermazione di una nuova etica del lavoro, conduce oggi a sancire un definitivo superamento della forma assunta dal lavoro nel novecento. La direzione in cui si orienta tale mutamento è ambigua, oscillando tra la sperimentazione di modelli di economia alternativa - che lanciano una sfida al capitalismo, come unico possibile modello economico - alla celebrazione di pratiche e modelli che configurano una sorta di scenario di "capitalismo bio- 
cognitivo" (Fumagalli 2017), che celebra l'estensione del capitalismo e l'affermazione della logica del profitto e del principio della competizione in ogni ambito della vita umana.

Entrambi gli scenari trovano nell'economia delle piattaforme un luogo privilegiato di realizzazione. L'apparente convergenza tra i due, in riferimento ad alcuni approcci, modalità organizzative e dinamiche di interazione, con particolare riguardo alla perdita di centralità delle strutture a favore delle relazioni orizzontali tra individui, contribuisce a generare confusione al momento di individuare una chiara linea di confine tra l'economia della collaborazione, definita in senso esigente, e una pluralità di pratiche che compongono il capitalismo delle piattaforme.

Qui giunge a compimento la trasformazione del modo in cui il lavoro viene pensato, organizzato, vissuto. Tale cambiamento è la complessa risultante delle trasformazioni dei modelli produttivi e di consumo, e procede in parallelo con l'evoluzione delle forme del capitalismo, associandosi a profondi mutamenti di tipo organizzativo, culturale e valoriale, trovando nelle piattaforme il terreno ideale di affermazione. Nell'ultimo decennio questi elementi trasformativi pregressi si sono saldati agli effetti della crisi economica, che, soprattutto nei paesi del sud Europa, hanno generato effetti negativi di medio e di lungo periodo nelle prospettive lavorative dei giovani.

Il passaggio dal fordismo al postfordismo, e quindi a un modello emergente, variamente definito capitalismo "cognitivo" (Vercellone 2006), "digitale" (Srnicek 2017) e delle piattaforme (Armano, Murgia 2017), è, in sintesi, accompagnato da una serie di trasformazioni in merito a:

1) Il mutamento nelle forme di regolazione, in direzione di una sempre maggiore destandardizzazione, pluralizzazione e frammentazione. Per anni si è parlato della crescente diffusione di contratti atipici, di tipo precario o parasubordinato, individuando una cesura tra insiders (i titolari di contratti di lavoro di tipo standard, a tempo indeterminato, oggetto di tutte le tutele conquistate nel corso del consolidamento dello Stato sociale) e outsiders (tutti coloro esclusi da questo tipo di inquadramento, e per questo meno tutelati). Tale dualizzazione del mercato del lavoro, negli anni, ha assunto una connotazione generazionale. Tale è, oggi, l'estensione del cosiddetto lavoro atipico, da suggerire la sua stessa ridefinizione come tale. Il lavoro frammentato, discontinuo, oscillante tra sotto-retribuzione ed elementi di gratuità, scarsamente (o per niente) tutelato finisce spesso per rappresentare l'unico modo di vivere l'esperienza lavorativa.

2) La fuoriuscita del lavoro dall'ambito lavorativo. Il lavoro si diversifica, negli orari e nei luoghi di lavoro, fino a con-fondersi all'interno della vita quotidiana. Ne risulta una progressiva indistinzione tra tempo di vita e 
tempo di lavoro. Se prima si lavorava per lo più nel proprio ufficio, in orari ben definiti, ora si lavora a casa, connessi con il proprio laptop in treno, in aeroporto, al tavolo di un caffè, a tutte le ore. Come fanno sempre più spesso i "nomadi digitali". Questa forma di sovrapposizione favorisce pratiche di (auto)sfruttamento e l'affermazione di nuove forme di sussunzione, ovvero di messa a valore delle relazioni e dei prodotti dell'azione umana, anche al di fuori dell'ambito lavorativo (Fumagalli 2017).

3) L'affermazione di una precarietà che assume sempre più una dimensione esistenziale, oltre che lavorativa, e che si estende alla sfera familiare, sociale e politica, oltre che economica. Come ci ricorda Rosa, se nell'era premoderna gli individui svolgevano per lo più lo stesso mestiere dei propri genitori, e nella modernità industriale svolgevano spesso un lavoro diverso da quello del padre, mantenendolo per tutta la vita, oggi lo stesso individuo sarà destinato a cambiare più volte lavoro nell'arco della sua vita, come esito del processo di "accelerazione sociale" (Rosa 2015). Il World Economic Forum, del resto, stima che il 65\% dei bambini di oggi svolgerà lavori che oggi non esistono. Ma, come è noto, la riformulazione degli strumenti di protezione sociale non ha seguito, se non in parte, il passo di questo mutamento profondo, con l'effetto di scaricare i rischi vieppiù dalle imprese - e dalle istituzioni pubbliche - ai singoli individui.

4) La ridefinizione del rapporto tra lavoro, cittadinanza e identità sociale. Il lavoro sempre meno si configura come un ambito distinto di azione, capace di definire e strutturare le forme della cittadinanza materiale, ruoli sociali, appartenenza di classe e stili di vita e habitus. Il lavoro contingente, frammentario, episodico, non produce quel tipo di identità sociale che caratterizzava invece l'operaio nella società industriale. Al punto di privare gli individui non solo della loro sicurezza economica ed esistenziale, ma anche di una definizione di sé.

5) Le trasformazioni nei rapporti tra lavatori e tra lavoratori e imprenditori. Imprenditori, lavoratori dipendenti, lavoratori autonomi, consumatori, figure un tempo distinte e riconoscibili, tendono oggi a sovrapporsi in modo ambiguo e ad interagire in forma innovativa, in direzione di un superamento dei ruoli e di una crescente ibridazione. Ciò crea, non di meno, aree ambigue, popolate da un numero crescente di free-lance, o di "pseudo-autonomi", che, di fatto, lavorano alle dipendenze di altri, ma al di fuori delle tradizionali tutele connesse allo status di dipendente. Anni fa ci si riferiva a questi soggetti come "autonomi di seconda generazione" (Bologna 1999). Oggi si tratta di una popolazione in espansione, che include i "padroncini" nella logistica, i fattorini (o riders, come vengono definiti oggi) di imprese come Foodora e Deliveroo. 
6) Il mutamento del rapporto tra esperienza individuale ed esperienza collettiva. Il lavoro sempre meno rientra in una narrazione collettiva, per diventare una questione privata. La frammentazione e la volatilità del lavoro conducono al superamento dell'elemento di fondo su cui si basava l'identificazione - e quindi l'azione collettiva - nel capitalismo industriale: la condivisione di un grande numero di lavoratori dello stesso contratto di lavoro, delle stesse mansioni, degli stessi orari, delle stesse condizioni. Oggi non solo gli individui "attraversano", senza radicarvisi, i luoghi di lavoro, ma spesso, negli stessi, convivono lavoratori con differenti inquadramenti contrattuali (contratti standard e non standard, collaboratori, dipendenti di imprese esterne e cooperative, per non parlare di tirocinanti e stagisti, pseudo autonomi, ecc). Infine, proprio la precarietà e la frammentazione diventano strumenti di un approccio competitivo nei confronti dei colleghi di lavoro, con l'adozione di una serie di strumenti che tendono a premiare chi è più disponibile alla deroga e, soprattutto, a punire chi esprime conflitto, o financo si attiva nel sindacato. Del resto, le tecnologie digitali rendono possibili nuovi, raffinati, strumenti di disciplinarmente (la geolocalizzazione, la tracciabilità costante delle attività e dei tempi con cui si realizzano le mansioni), che permettono un controllo totale sul lavoratore e inibiscono ulteriormente, oltre ai suoi margini di autonomia, la possibilità di sviluppare strategie comuni con gli altri lavoratori.

7) La ridefinizione del rapporto tra formazione e lavoro. Un tempo rigidamente definite, e legate all'articolazione delle fasi di vita, queste due fasi sempre meno diventano separate per divenire sempre più "alternate" e interconnesse, in virtù del principio del life-long learning e dei principi, a questo collegati, dell'attivazione, dell'occupabilità, ecc. L'introduzione dell'alternanza scuola-lavoro obbligatoria, in Italia, con la L. 107/2015, sancisce l'istituzionalizzazione di questo tipo di approccio.

\section{NON SOLO SHARING ECONOMY: I TANTI VOLTI DEL CAPITALISMO DIGITALE}

L'economia della condivisione (Sharing Economy) può essere definita, in prima approssimazione, come un nuovo modello economico in cui la centralità del possesso è sostituita dalla condivisione e dal riutilizzo. Il concetto è stato sottoposto a critica da parte della letteratura scientifica più recente, per via della sua indeterminatezza, e perché tende a riunire sotto lo stesso ombrello sia pratiche "dal basso", orientate socialmente e ispirate a costruire modelli economici alternativi a quello capitalista, e pratiche verticistiche, gestite in forma privatistica e orientate al profitto. Si tende dunque a privilegiare il concetto - più circoscritto - di economia della collaborazione. Quest'ultimo, allo spostamento dell'enfasi dal possesso all'accesso (Rifkin 2001), ne aggiunge una altrettanto forte 
sulla valorizzazione della dimensione relazionale e comunitaria. L'idea di fondo che suggerisce questo spostamento semantico è che il vero valore aggiunto di queste esperienze, sul piano della rielaborazione del rapporto tra economico e sociale, non si generi nella condivisione - elemento comune in molte pratiche orientate al profitto - ma proprio nella valorizzazione dell'aspetto della collaborazione: una collaborazione che non è meramente focalizzata sull'oggetto dello scambio, ma che coinvolge la stessa relazione tra gli individui, che non si limita cioè ai mezzi ma si estende ai fini (Sundarajan 2015), trasformando gli utenti in una vera comunità (Iaione 2016).

L'economia della collaborazione, d'accordo con Alaminos e Penalva, descrive "una nuova maniera di strutturare le relazioni economiche (che) conduce, potenzialmente, alla reinvenzione dei legami sociali (...) includendo elementi di valorizzazione dell'interscambio che vanno al di là dell'ottenimento di benefici economici: ottenere un valore sociale, coesione, integrazione, formazione, benessere, felicità, ecc, equilibrio con l'ambiente e utilizzo di risorse locali" (Alaminos, Penalva 2018). A questo nuovo tipo di relazione economica si attribuisce una serie di virtù, di tipo sociale, civico, culturale, economico, ma anche ambientale (Zvolska, 2015), dal momento che lo scambio e la condivisione favoriscono «la diffusione di pratiche dirette a sfruttare al meglio la funzionalità di beni che hanno una capacità in eccesso rispetto all'uso individuale», creando per questa via anche nuove opportunità di mercato e quindi lavorative (Fondazione Unipolis, 2015, p. 18). L'economia della collaborazione, idealmente, si nutre - e contribuisce ad alimentare - la fiducia tra estranei, tende alla massimizzazione delle risorse, valorizza la massa critica, e contribuisce a mettere al centro l'idea di bene comune (Botsman e Rogers, 2010).

Ma da dove trova impulso questa reinvenzione dell'agire economico? A ben vedere, molte delle pratiche che compongono questo modello precedono la nascita e lo sviluppo delle tecnologie digitali, e in molti casi la stessa affermazione dell'economia capitalista. Anzi, rappresentano un recupero di pratiche proprio del periodo precedente il consolidamento della modernità industriale e dello Stato sociale. Secondo Pais e Mainieri (2015), alle radici delle nuove pratiche economiche non vi è soltanto l'incontro tra diffusione dei media digitali ed effetti congiunturali della crisi economica, ma un "ripensamento strutturale dei rapporti tra economia e società, basato sulla creazione di legame sociale come fondativo dello scambio economico". In chiave polanyana, questo ripensamento può essere concepito come l'affermazione di un nuovo equilibrio tra tre forme di regolazione: lo scambio (che nell'economia capitalista diventa la forma di azione economica ampiamente predominante), la reciprocità (tipica dell'agire prosociale) e la redistribuzione (tipica dell'intervento pubblico). L'economia della collaborazione si genera così come una forma intermedia tra scambio e reciprocità, poiché l'agire in rete permette di realizzare un progetto che porterà 
benefici individuali a tutti gli individui coinvolti, mentre l'economia della condivisione - limitatamente alle sue espressioni meno market-oriented rappresenterebbe più una forma intermedia tra reciprocità e redistribuzione, poiché ne sono protagonisti un gruppo di individui che mette in comune le risorse per produrre un bene o un servizio utile per l'intera comunità.

L'utilizzo dei media digitali è fondamentale per supportare relazioni sociali a distanza, in cui la prossimità sociale non coincide con quella geografica, che si fondano sulla disintermediazione, sulla orizzontalità e sul superamento della distinzione tra produttore e consumatore, e in cui diventa centrale la risorsa della reputazione. Alla base dell'economia collaborativa (Kostakis e Bauwens 2014) vi è, infatti, l'attivazione di reti disperse formate da individui e comunità connesse, che pone in secondo piano il ruolo delle istituzioni centralizzate (Botsman, 2013).

Le tecnologie si pongono a fondamento di pratiche di innovazione sociale, perseguendo una "nuova forma di integrazione tra società ed economia in cui le relazioni sociali diventano il motore non solo di coesione sociale, bensì di sviluppo economico basato sulla creazione di nuove forme di comunità", e hanno un esplicito orientamento sociale, adottano modelli di governance cooperativa e forme di gestione collettiva, collaborativa, aperta (Regione Toscana 2017).

Ma quanto le pratiche concrete che solitamente sono associate alle etichette di economia della condivisione, o di economia della collaborazione, effettivamente aderiscono a questo modello?

A ben vedere, le esperienze che nell'immaginario collettivo sono comunemente associate alla Sharing Economy - ci riferiamo a AirBnB, la piattaforma di condivisione di camere e appartamenti, e a Uber, la piattaforma che rende possibile l'incontro tra offerta e domanda di trasporto con conducente, rivelano un prevalente orientamento di tipo economico, ovvero di massimizzazione del beneficio economico da parte di chi offre un bene/servizio e di chi ne usufruisce, in un contesto in cui l'intermediazione è gestita da un'impresa privata che realizza profitto e che, per di più, manifesta una evidente tendenza alla costruzione di un oligopolio. Uber è un esempio di On-Demand Economy, in cui, come si approfondirà nel paragrafo successivo, l'utilizzo delle APP permette modalità innovative di incontro tra la domanda e l'offerta nel settore di servizi tradizionali. La dimensione relazionale e l'attenzione alla sostenibilità non appaiono più spiccate che nel più tradizionale servizio di Taxi (in cui, al pari, il cliente dialoga con l'autista e contribuisce a non ingolfare le strade con le auto private), se non ché perché, assai di più che nel servizio di taxi, i meccanismi di valutazione spingono l'autista a rivelarsi accogliente e affidabile, sul piano relazionale, oltre che della prestazione professionale in sé. 
AirBnB, da parte sua, è il più celebre esempio di Peer-to-Peer Economy, il cui ricorso, da parte di chi affitta e di cerca casa, è di norma fondato su logiche economiche, o comunque su motivazioni che esulano dall'aspetto della relazionalità e della sostenibilità. La componente relazionale, ancor più dal momento che diventa un fenomeno di massa, diventa residuale rispetto ad altri criteri, nel determinare la scelta dei proprietari di affittare sulla piattaforma e della decisione dei clienti di orientarsi su questa, invece che su un albergo. Al di là della retorica sul vivere in maniera diversa l'esperienza del viaggio, chi affitta l'appartamento su AirBnB è spinto dalla possibilità di realizzare profitti maggiori rispetto all'affitto tradizionale, al punto che la diffusione degli affitti turistici a breve periodo, in molte città, amplifica i pregressi processi di gentrification, fino a generare una vera e propria espulsione degli abitanti dalle città (cfr. Raffini 2016). Al pari, chi si rivolge a AirBnB lo fa di norma per risparmiare, oltre che per vivere in modo diverso l'esperienza turistica. Altre esperienze fondate sula condivisione di beni e servizi, pensiamo alle forme più comuni di car-sharing, gestite da imprese private, rientrano più nella Rental-Economy che nell'economia della collaborazione.

Tutte quelle esperienze che utilizzano le piattaforme digitali, prevedono forme di condivisione, e valorizzano in alcun modo la relazionalità orizzontale tra gli utenti, ma non rientrano in una definizione stringente di economia della collaborazione.

Rientriamo nell'abito dell'economia della condivisione solo se vi è una centralità della componente relazionale, all'interno di una reale prospettiva di orizzontalità e di reciprocità, e in presenza di un orientamento prosociale, o comunque volto ad affermare relazioni economiche alternative a quelle di tipo capitalista. In questo caso si configura un inedito incrocio tra scambio, reciprocità e redistribuzione, ovvero si sviluppano inedite connessioni tra agire economico ed agire sociale, in direzione di una ri-connessione dell'economia nel sociale (Pais, Provasi 2015), che trova espressione in pratiche di produzione collaborativa, di consumo collaborativo, di apprendimento collaborativo, di finanza collaborativa e di governance collaborativa. Queste, insieme, possono essere considerate come i "cinque pilastri" che compongono l'economia collaborativa (Fondazione Unipolis, 2015, p. 16).

Sul piano pratico, rientrano in questa cornice una pluralità di esperienze che spaziano dal Crowd-Funding, utilizzato per reperire risorse per realizzare progetti di vario tipo, al co-working, in cui lavoratori altrimenti isolati si condividono spazi, strumenti, ma anche occasioni di conoscenza, di confronto e di collaborazione. Si tratta di pratiche accomunate dal superamento delle tradizionali impostazioni gerarchiche e verticistiche, da una ridefinizione, in chiave orizzontale, dei ruoli, tesa a valorizzare il principio della collaborazione, 
al servizio della creatività e dell'innovazione, da una tendenza diffusa a ibridare contesti e orientamenti all'azione. Sul piano dell'etica del lavoro, l'orientamento strumentale è, almeno sul piano dei discorsi, intrecciato a un orientamento prosociale, che trova espressione nel perseguimento della sostenibilità sociale ed ambientale.

\section{VERSO UNA NUOVA ECONOMIA?}

La centralità della valorizzazione del singolo, della connessione e della reticolarità, della connessione e dell'orizzontalità, perseguite in campo economico e lavorativo attraverso l'affermazione di questi nuovi orientamenti, può essere considerata il corrispettivo di quanto avviene in ambito politico, con la crisi delle organizzazioni politiche tradizionali, l'affermazione di nuove modalità organizzative a rete, la celebrazione della centralità dell'individuo e delle sue cangianti forme di connessione pubblica. Sullo sfondo vi sono, in generale, i processi di deistituzionalizzazione, che trovano espressione in una inedita centralità del rapporto diretto tra individui, bypassando le istituzioni di mediazione. Ciò avviene tanto nelle nuove forme di produzione tanto nelle forme di consumo, il cui protagonista è la figura del prosumer.

L'affermazione di nuovi principi e valori e lo sviluppo di nuove dinamiche organizzative e relazionali trova espressione in nuove filosofie imprenditoriali e di marketing, che si fondano sulla celebrazione di un rapporto - suppostamente - orizzontale e collaborativo, non solo all'interno dell'impresa, ma tra imprese e consumatori. Uno degli elementi di fondo che garantisce questo tipo di rapporto è l'utilizzo innovativo delle ICTs come strumento di connessione continua e orizzontalizzante.

Una delle espressioni di questo nuovo modo di concepire il ruolo dell'impresa è l'affermazione, in vece del tradizionale concetto di marketing, di quello di societing (Fabris 2008). Con questo si indica un nuovo modo di concepire l'impresa, in cui principi e valori che muovono le strategie aziendali non sono più il mercato ma la società nel suo complesso. Ciò in rispetto al principio per cui tra mercato e altre sfere della società - e così tra azione economica e altre forme di azione, non vi è un rigido steccato. Al centro del paradigma del societing vi è l'apertura verso l'esterno, l'assunzione di nuove responsabilità sociali, etiche ed ambientali, il riconoscimento di nuove forme di socialità, per lo più mediate dalle tecnologie, la centralità del concetto di relazione al posto di quella di transazione, un modo di guardare al cliente che lo trasforma in partner e co-produttore. Il concetto di consumatore quale al contempo produttore, si lega alla visione del consumatore come "consumattore", che pone al centro del rapporto con l'impresa e, in generale, delle relazioni economiche, non più un soggetto disincarnato, che partecipa allo scambio nel suo ruolo di consumatore, ma un ruolo attivo di 
soggetto, che coinvolge ogni sua dimensione. Per Arviddson e Giordano (2013), il paradigma del societing si pone al centro di un cambio di paradigma organizzativo, veicolante nuovi modi di pensare e praticare l'impresa, che recepiscono i nuovi valori diffusi e che offrono gli strumenti per valorizzare la produzione condivisa, uscendo da una logica di mercato di massa. Tale cambiamento di paradigma, si sostiene, rappresenta il presupposto per uscire dalla crisi economica e sociale. La prospettiva di fondo in cui si colloca il paradigma del societing è quella dell'innovazione sociale (Murray et al. 2012), i cui principi chiave sono interazione, innovazione e collaborazione. L'utilizzo delle tecnologie assume qui un ruolo centrale nel coinvolgimento dei destinatario di un prodotto o di un servizio già al momento della sua progettazione e nel porre al centro il "forte uso di networks ramificati per sostenere e gestire le relazioni, aiutati da ogni forma possibile di comunicazione dei confini sfuocati tra produzione e consumo" (ivi). Seguire questo tipo di approccio significa riconoscere al consumatore un orientamento che non è prettamente modellato da motivazioni utilitaristiche, ma da spinte di natura etica, richiedendo all'impresa, a sua volta, di non limitarsi ad operare su un mercato, da di inserirsi positivamente nel contesto sociale.

Fin qui si è visto come le tecnologie si pongono al servizio di una trasformazione delle relazioni economiche, sul piano degli approcci, degli orientamenti valoriali e dei modelli organizzativi, che promuove processi di innovazione al contempo tecnologica e sociale che, nel loro complesso, convergono nel promuovere un cambio di paradigma, al cui centro vi è l'integrazione tra orientamento economico e orientamento sociale, il coinvolgimento dei destinatari, la centralità della dimensione relazionale, lo sfocarsi della distinzione tra produttore e consumatore. L'ibridazione tra ambiti e orientamenti all'azione, in questo caso, prende la forma di una "civilizzazione dell'economia" (cfr. Zamagni 2004), e favorisce l'affermazione dell'impresa sociale, ovvero di un approccio all'impresa - e al lavoro - che integra e concilia, in maniera costitutiva, un approccio imprenditoriale, orientato cioè alla sostenibilità economica e alla produzione di lavoro, al perseguimento di una funzione sociale. Ciò significa che non solo l'agire imprenditoriale si realizza senza produrre esternalità negative, ma che si pone l'obiettivo esplicito di produrre esternalità positive, ovvero benefici per la società e per la comunità nel suo complesso, e non solo per l'impresa stessa.

L'innovazione sociale prende dunque forma lungo un continuum che comprende, da una parte, una serie di pratiche di economia alternativa (pensiamo alle esperienze di riuso e di condivisione, alle banche del tempo, alle social streets, a una pluralità di esperienze di mutualità che trovano nell'utilizzo delle piattaforme digitali lo strumento per ampliarsi, espandersi qualitativamente e quantitativamente, raggiungendo inedite dimensioni di scala), e dall'altra la costruzione di modi diversi di pensare e di organizzare 
l'impresa e di definire il suo ruolo nella società. In questo senso possiamo fare riferimento a quella che è stata definita "industria 4.0" (CGIL Lombardia 2017). Questa, frutto della quarta rivoluzione industriale, fondata sulla virtualizzazione - e sulla sovrapposizione tra mutamento tecnologico e mutamento culturale - è un'impresa sempre più automatizzata, "smart" e interconnessa, che si fonda su una comunicazione multidirezionale, dinamica e costante tra tutti gli attori e un loro coinvolgimento attivo; che denota una spiccata capacità di riflessione strategica e di adattamento flessibile; che si caratterizza per un costante e pieno utilizzo delle tecnologie digitali ${ }^{1}$. Un altro concetto fondamentale per comprendere le trasformazioni dell'agire economico è quello di impresa sociale, concetto che definisce "organizzazioni produttive che perseguono in modo esplicito non il profitto, ma il soddisfacimento dei bisogni delle persone e della collettività, e quindi non distribuiscono utili, o ne distribuiscono una parte limitata e sono controllate dagli stessi beneficiari" (Borzaga e Fazzi 2011, 16). Le imprese sociali sono imprese, nel senso che producono in forma continuativa e professionale beni o servizi per la collettività, si fondano su personale retribuito. L'obiettivo primario, tuttavia, è quello che definisce il non profit, ovvero quello di offrire risposte a bisogni sociali. La finalità dell'imprenditore sociale non è il solo perseguimento del successo economico, ma il conseguimento di un impatto positivo sulla società. La dimensione imprenditoriale è definita dalla produzione continuativa e professionale di beni o servizi, da una elevata autonomia nella costituzione e nella gestione, dall'assunzione di un rischio di impresa, dalla presenza di lavoratori retribuiti, che affiancano eventualmente i volontari. La dimensione sociale è invece definita dagli obiettivi sociali dell'attività imprenditoriale, dal suo essere un'impresa collettiva, e non individuale, dalla partecipazione degli stakeholders e dalla non redistribuzione degli utili (Borzaga e Zandonai 2009). L'impresa sociale realizza un'ibridazione tra linguaggi e forme di azione che trasforma tanto l'azione economica tanto l'azione solidale, non in direzione di un "assorbimento" o di una "sottomissione" di una all'altra, ma della costruzione di una forma di azione inedita che porta a compimento il processo di de-differenziazione proprio della tarda modernità e rappresenta la cristallizzazione di nuove combinazioni tra distinte logiche di azione. Rappresenta una contaminazione tra agire razionale rispetto allo scopo, tradizionalmente associato all'azione economica, e agire razionale rispetto al valore, tradizionalmente appannaggio dell'agire solidale. L'impresa sociale concilia sostenibilità economica e sociale, ponendo l'enfasi principale sulla

\footnotetext{
${ }^{1}$ Secondo McKinsey, queste ultime interverranno sempre più trasformando il volto dell'impresa in quattro ambiti: big data, open data, e cloud computing; analytics (produzione di valore a partire dall'elaborazione dei dati); interazione tra uomo e macchina; connessione costante tra digitale e "reale". Insieme, questi elementi definiscono la capacità di un'impresa di profilarsi come una Smart Factory che pone al centro il principio della collaborazione, sia tra operatore, macchine e strumenti (Smart Production), sia tra impresa, fornitori, collaboratori e clienti (Smart Services) e che è orientata all'utilizzo della Smart Energy, in un'ottica di sostenibilità ambientale.
} 
seconda, ma individuando nella prima il presupposto su qui fondare quest'ultima. Si conforma ai principi e ai meccanismi che regolano il mercato, sul piano delle modalità operative, ma dall'economia di mercato non mutua il principio della massimizzazione e dell'introitazione privata del profitto, perseguendo, al contrario, un principio di redistribuzione. In questo modo si capovolge uno dei principi basilari dell'economia di mercato, che attribuisce alle imprese private la massimizzazione dei profitti e la distribuzione delle esternalità negative poiché, al contrario, l'impresa sociale si pone l'obiettivo di produrre esternalità positive e non si pone come obiettivo prioritario quello di generare profitto.

La Commissione Europea individua nella diffusione dell'impresa sociale un fondamentale veicolo di innovazione sociale, capace di fondare un modello di sviluppo che integri virtuosamente obiettivi sociali, comunitari e ambientali, contribuendo per questa via al raggiungimento degli obiettivi di sviluppo della UE contenuti nella strategia EU2020: costruire società coese, competitiva, fondate sulla conoscenza e orientate alla sostenibilità sociale ed ambientale. A tal fine, la CE approva, nel 2011 (Commissione Europea 2011), la Social Business Initiative, il cui obiettivo è creare un terreno fertile per lo sviluppo dell'impresa sociale in Europa, identificando nell'inserimento lavorativo, nei servizi alla persona, nello sviluppo territoriale, alcuni ambiti centrali di applicazione.

\section{IL LAVORO NELL'ECONOMIA DELLE PIATTAFORME}

Si è evidenziato come il mutamento tecnologico possa porsi a servizio di pratiche di innovazione economica e sociale, che attribuiscono nuovi significati e favoriscono nuovi approcci al lavoro in direzione di un superamento della forma novecentesca dello stesso.

Riteniamo ora da approfondire un'altra forma in cui si esprime la trasformazione del lavoro nell'economia elle piattaforme, in direzione, al contrario, di un ritorno a condizioni e pratiche tipiche del primo capitalismo industriale, quello precedente i "gloriosi trent'anni", e quindi l'estensione dei sistemi di tutela dei lavoratori e dei diritti sociali.

Si tratta di fenomeni che, a ben vedere, caratterizzano il lato oscuro dello sviluppo dell'economia delle piattaforme, concetto che descrive una rivoluzione delle dinamiche di produzione e di consumo reso possibile dall'utilizzo dei nuovi media.

Accompagnata, inizialmente, dalla celebrazione delle sue capacità di generare effetti virtuosi, di promuovere la cooperazione, di favorire l'ingresso in un mondo "smart", guidato dall'affermazione di una nuova "classe creativa" (Florida 2002), rivela oggi essere il terreno di sviluppo di pratiche che esacerbano le 
dinamiche di dualizzazione del mercato del lavoro, ovvero il crescente divario tra una minoranza di lavoratori coinvolti in impieghi altamente qualificati, professionalmente, personalmente ed economicamente gratificanti, e una maggioranza di lavoratori precari, intermittenti, sottoretribuiti e debolmente tutelati. Di offrire, per questa via, gli strumenti per completare la frammentazione, la deregolamentazione e la mercificazione del lavoro, e le forme di sfruttamento a queste connesse, al punto di prefigurare - e non solo nei lavori con debole qualificazione - il ritorno a forme più o meno velate di sfruttamento, come il cottimo.

Lo scenario utopico, in cui il progresso tecnologico, l'aumento della produttività, e il progressivo shift verso il settore dei servizi e della conoscenza, avrebbe "liberato" gli uomini dal lavoro, nel senso che avrebbe reso possibile una diminuzione complessiva del tempo lavorativo, nel quadro di un miglioramento delle condizioni di vita di e redistribuzione della ricchezza, sembra oggi rovesciarsi in uno scenario distopico, in cui si lavora di più, in forma sempre meno regolata e tutelata, e si è retribuiti meno. Più che alla "fine del lavoro", si assiste alla fine del lavoro salariato e accompagnato dai diritti e dalle tutele conquistate dai lavoratori nei "trenta anni gloriosi". Le ore di lavoro e, nel complesso, la parte di vita messa a valore, aumentano, mentre diminuiscono tutele, diritti, retribuzione. Ciò perché si estende, fino a coprire potenzialmente ogni ambito di vita, l'ambito in cui produciamo valore, in forma diretta o indiretta (producendo contenuti, ampliando le proprie reti e le proprie competenze, stabilendo connessioni, tramite il coinvolgimento in attività che arricchiscono il proprio $\mathrm{cv}$ ), ma il valore prodotto è per lo più messo a profitto da altri. $\mathrm{O}$ il corrispettivo ricevuto in cambio del lavoro, come si approfondirà, è "pagato" in modalità diverse: con una promessa di lavoro, con l'impiegabilità, con il miglioramento del proprio cv. Per comprendere in che modo l'economia delle piattaforme, fondata sull'utilizzo dei media digitali per creare nuove forme di organizzazione del lavoro, di produzione e di erogazione di beni e servizi, contribuisce a generare processi di segno opposto - e di amplificare, per questa via, il divario tra inclusi ed esclusi, "salvati" e "sommersi", vale la pena procedere a un tentativo di tassonomia delle forme pratiche in cui si manifesta: economia della collaborazione, economia della condivisione, GIG economy, per soffermare, infine, lo sguardo su alcuni scenari di sviluppo virtuoso. Ciò infatti, a nostro parere, permette di indagare da una prospettiva privilegiata, il modo in cui dietro all'etichetta di "economia dei servizi" - in generale, e più nello specifico di "economia delle piattaforme", si celino destini lavorativi assai diversificati, che comprendono i top-manager della finanza, i creativi dell'industria culturale, gli imprenditori high-tech, ma anche i lavoratori dei servizi a bassa qualifica, gli operatori dei call centres, fino agli operatori che si pongono ai livelli più bassi delle imprese della Crowd-Economy o dell'On Demand Economy, che corrispondono, come vedremo, ai lavoratori pagati a cottimo per realizzare "task" 
estremamente parcellizzati, attraverso la rete, o ai lavoratori che prestano i loro servizi ai clienti attraverso la mediazione di App, come gli autisti di Uber o i riders di Foodora, di Deliveroo, di Glovo, di Just Eat, ecc. In mezzo alle due fattispecie, ve ne è una terza, probabilmente la più affollata. È quella che corrisponde ad una quota crescente di lavoratori che, spinti dall'ambizione di perseguire una carriera lavorativa gratificante - o almeno dignitosa - accettano impieghi sottoretribuiti, sottotutelati, spesso sottoqualificati. Questa condizione, che si dipana attraverso contratti precari, pseudo-autonomi e che sovente contempla forme più o meno esplicite di lavoro gratuito e di (auto)sfruttamento, inizialmente percepita come transitoria, sempre più rischia di diventare strutturale. Prelude non a un futuro da lavoratore qualificato, gratificato e ben retribuito, ma a una carriera da lavoratore posto ai gradini più bassi - o perennemente sottoposto al rischio di scivolarvi - dell'economia digitale.

Ciò ci spinge a vedere nel capitalismo digitale - o delle piattaforme - come a una nuova fase del capitalismo che, rispetto alla precedente, presenta opportunità e rischi e che, soprattutto, richiede un ripensamento radicale del rapporto tra lavoro e società e dei diritti.

In prima approssimazione, possiamo definire l'economia delle piattaforme come un nuovo modello economico che utilizzano le tecnologie digitali per facilitare le relazioni, in termini di scambio o di cooperazione tra le persone, che apre la strada a cambiamenti radicali nel modo in cui lavoriamo, socializziamo, creiamo valore economico e competiamo per i profitti (Kenney, Zysman 2016). Sotto l'ombrello dell'economia delle piattaforme - Platform Economy - possiamo quindi distinguere due fenomeni distinti: il Platform Capitalism e il Platform Cooperativism (Passoni 2015). Il primo definisce un modello di capitalismo che ha per oggetto la vendita o il noleggio/affitto di risorse, e che mette a valore le relazioni peer-topeer degli individui coinvolti (o, nella variante del Labour Platforms, organizza l'erogazione di "task" lavorative, cfr. Farrell e Greig 2016). Al contrario, il concetto di Platform Cooperativism definisce un modello alternativo di interazione, fondato su reciprocità e mutualismo. In questo, le potenzialità offerte dalla rete in termini di nuove possibilità di interazione, di condivisione, di produzione collettiva, di collaborazione, non sono appropriate a scopo di profitto dai proprietari delle piattaforme, ma sono sfruttate per generare processi di innovazione sociale. Le esperienze e le pratiche che rientrano nel modello del Platform Capitalism - che definiscono cioè un nuovo modo di realizzare profitto, attraverso i media digitali - a ben vedere, sono assai più diffuse, sul piano dell'impatto effettivo, di quelle che possono essere fatte rientrare nel Platform Capitalism. Basti pensare alle esperienze più note, e che sono spesso sbrigativamente associate a concetti come Sharing Economy, come Uber o AirBnB. Quest'ultima, per esempio, è una piattaforma che utilizza le potenzialità di disintermediazione offerte dalla rete per consentire ai proprietari di appartamenti di trarvi un profitto, superiore a quello ottenibile tramite l'affitto tradizionale di lungo periodo, agli affittuari di 
risparmiare e, soprattutto, ai proprietari della piattaforma di ottenere straordinari profitti, oggi assai superiori a quelli delle principali catene alberghiere, senza possedere appartamenti e con un numero di dipendenti molto ridotto. In questo caso, la dimensione dell'incontro, o della condivisione, appare di fatto assai ridotta, mentre sono da tenere in seria considerazione gli impatti di questo tipo di pratiche in termini di perdita di posti di lavoro e di creazione di posti di lavoro de-regolamentati, di gentrification, dal momento che la possibilità di ottenere maggiori guadagni con gli affitti turistici spinge a un allontanamento degli abitanti dai quartieri centrali (cfr Raffini 2016). Il modello economico di Airbnb e di Uber, due società che con le loro App e le loro piattaforme digitali sono entrate nell'immaginario sociale come "modello vincente" di Sharing Economy, in realtà ricade pienamente nel perimetro dell'economia tradizionale e, dunque, nelle logiche dello scambio di mercato.

Lo stesso può essere detto in riferimento a piattaforme con TaskRabbit, che consentono l'incontro tra domanda e offerta per la realizzazione di una varietà di prestazioni (dalla riparazione della caldaia alla manutenzione del giardino) o come le svariate piattaforme di consegna a domicilio. In tutte queste non siamo di fronte a forme di condivisioni o di riutilizzo - e tantomeno a modalità di scambio che prevedono una spiccata componente relazionale ma, meramente, di fronte a modalità estremamente efficienti di organizzazione dell'impresa, in cui il profitto si fonda sulla messa a sistema di un sistema di lavoro "freelancizzato", il cui costo si scarica completamente sui lavoratori stessi, che agiscono in modo flessibile, intermittente, parcellizzato, con meccanismi di protezione molto ridotti, se non assenti.

Il capitalismo delle piattaforme ha un evidente impatto trasformativo rispetto ai modelli tradizionali di impresa, sia sul piano del lavoro sia sul piano del consumo, con una netta tendenza a rendere porosi i confini tra i due ambiti. L'estrazione di valore e la creazione di profitto avvengono nelle due dimensioni e nella loro ibridazione. Sul piano del consumo, dove i protagonisti sono i prosumers, al tempo stesso produttori e fruitori, il capitalismo delle piattaforme crea profitto mettendo a valore i big data, ovvero gli enormi archivi costruiti profilando le attività in rete degli utenti e utilizzando le informazioni da loro fornite, intenzionalmente o meno. Per alcune, come Amazon o Netflix, l'utilizzo dei big data è a supporto dell'attività di vendita di prodotti. Consente di proporre al cliente - incentivandolo alla fruizione e al consumo - i prodotti che più rispondono ai suoi gusti e ai suoi interessi, perché simili ai consumi precedenti o perché rispondenti al suo profilo di utente. Per altre, come Facebook e Google, la cui maggioranza degli utenti usufruisce di servizi gratuiti, l'utilizzo dei big data genera la parte principale del profitto: queste imprese utilizzano, infatti, le informazioni e i contenuti prodotti dagli utenti per venderli a terzi, oltre che per offrire all'utente stesso un servizio o un prodotto profilato (Passoni 2016). Nel 2018, la notizia che Facebook ha venduto a Cambridge Analytics i dati di 
centinaia di migliaia di utenti, e che questi dati siano stati usati a fini elettorali, ha alimentato polemiche e provocato un crollo delle quotazioni in borsa dell'impresa californiana, ma è lo stesso modello di business che, a ben vedere, si fonda su questo tipo di pratiche: la logica "estrattiva" del capitalismo delle piattaforme (Vecchi 2017) si afferma dal momento che sancisce lo scambio tra l'uso (apparentemente) gratuito delle piattaforme e la cessione dei propri dati personali per fini di profitto dei proprietari delle piattaforme. E' così che, agendo da prosumer, creiamo valore per le piattaforme che utilizziamo, e che fanno sì che, consultando l'home page di un quotidiano, ci appaia non più una pubblicità standard, ma l'invito ad acquistare un prodotto che rispecchia il nostro profilo e i nostri stili di consumo. Magari quel paio di scarpe che avevamo guardato la sera prima, che ci erano piaciute tanto, e che per averle basta cliccare con il mouse e inserire la carta di credito. Anche mentre vado in metro al lavoro. Anche dal cellulare mentre aspetto un amico fuori dal bar. I meccanismi di base che si pongono a fondamento della creazione di valore a partire dalle nostre interazioni in rete - e che spiegano l'apparente gratuità delle piattaforme, sono la personalizzazione pubblicitaria e la bolla dei filtri. Quest'ultima espressone descrive il modo in cui gli algoritmi delle piattaforme digitali tengono memoria, delle nostre ricerche, dei nostri percorsi di navigazione, dei nostri acquisti e li incrociano con i dati che noi stessi forniamo per accedere ai servizi, per proporci acquisti rispondenti ai nostri gusti e per metterci in contatto con persone che la pensano come noi, con evidenti ricadute, anche sul piano sociale, politico e culturale.

Ma non tutte le espressioni del capitalismo delle piattaforme si basano sulla messa a valore delle informazioni e dei contenuti prodotti dagli utenti. Molte altre si fondano su una più tradizionale erogazione di lavoro, da parte di lavoratori che, non di meno, sempre meno sono riconosciuti come tali, e che vivono profondi processi di mercificazione, di sfruttamento e di negazione dei diritti. Parliamo, in questo caso, di Crowd Capitalism e di On Demand Economy (De Stefano 2015), le due forme con cui si presentano le Labour Platforms, e che "descrivono attività economiche basate sull'utilizzo di piattaforme internet che permettono l'incontro tra utilizzatore che richiede un bene o un servizio e un altro che è in grado di fornirlo "condividendo" il patrimonio di beni, competenze, tempo di cui è in possesso": si tratta di pratiche cui ci si riferisce spesso facendo riferimento al concetto di Sharing Economy. Ma, a ben vedere, con quest'ultima condividono i presupposti tecnologici e buona parte delle modalità di espressione, ma non l'orientamento relazionale e sociale. Nonostante la condivisione di molte caratteristiche - i due modelli sono dunque da mantenere concettualmente distinti (Dagnino 2015), mancando nei primi quella dimensione di incontro tra dimensione economica e sociale, in direzione di una "civilizzazione dell'economia", che, teoricamente, caratterizzerebbe l'economia della condivisione. 
Il Crowd Capitalism, nello specifico, definisce quegli ambiti in cui le piattaforme digitali permettono di sostituire il ricorso al lavoro salariato, o al professionista, nelle forme tradizionali, con meccanismi di incontro online tra domanda e offerta, su micro-task che vengono realizzati "in remoto", ovvero possono essere realizzati con il proprio computer, senza necessità di spostarsi. Non è necessario che vi sia una relazione tra chi offre e chi cerca lavoro, poiché l'incontro avviene mediante la piattaforma, e ha per oggetto prestazioni che possono essere effettuate dal proprio computer, a casa, e quindi da ogni parte del mondo. Il lavoro è disperso tra una folla di potenziali lavoratori che sono disponibili a effettuare una serie di microlavori, in condizioni di isolamento, frammentazione e concorrenza. Esteso anche a mansioni che richiedono una media o alta qualifica, vi rientrano traduzioni, revisione di bozze, grafica, programmazione, è chiaro l'effetto di rincorsa al ribasso che si ha, dal momento che il lavoratore europeo o nordamericano si contende i micro-task con i lavoratori indiani o pakistani. La On Demand Economy, al contrario, descrive modelli organizzativi in cui la realizzazione di prestazioni lavorative viene completamente ristrutturata grazie ad un intenso utilizzo delle piattaforme digitali. Le piattaforme, di norma attraverso App, rendono possibile l'incontro tra domanda e offerta, in relazione a una molteplicità di servizi che spaziano dalle consegne di cibo, all'estetica e alla manicure, ai lavori domestici, fino al lavaggio dell'auto. Tablet e Smartphone diventano vere e proprie "infrastrutture invisibili" che connettono imprese, clienti e lavoratori altamente mercificati, permettendo un massimo livello di flessibilità (Aloisi 2016). L'incontro tra domanda e offerta non si completa all'interno della piattaforma, ma con l'erogazione di un servizio che prevede un incontro fisico tra lavoratore e destinatario dello stesso. La piattaforma che permette l'incontro tra domanda e offerta ottiene una percentuale per ogni transazione, mentre i lavoratori che la effettuano non sono dipendenti della piattaforma ma agiscono come lavoratori suppostamente autonomi, e sono inoltre incentivati ad offrire un servizio di qualità a prezzi bassi, per mantenere alto il proprio ranking.

I due modelli riconfigurano in modo radicale il rapporto tra impresa, lavoratori e fruitori dei servizi, per lo più in direzione di un peggioramento delle condizioni di lavoro, tanto da convergere in quella che è stata definita GIG Economy, traducibile in italiano con "economia dei lavoretti" (Staglianò 2018). Il lavoro prodotto nell'ambito della GIG Economy molto poco si conforma alle retoriche dei lavori cognitivi, altamente gratificanti, promessi dalla società della conoscenza, e che, piuttosto, configurano una sorta di "taylorismo digitale" (Formenti 2011; Regini 2012), i cui lavoratori, messi a margine del sistema di welfare, per alcuni aspetti assumono tratti tipici dell'operaio di massa del fordismo, e per altri caratteri tipici della fase precedente al fordismo, in termini di sfruttamento, alienazione, mercificazione -a proposito, Berg e De Stefano (2018) suggeriscono 
di guardare alla rivoluzione digitale come un grande fenomeno di "rebranding" del lavoro come merce (cfr. anche Ciccarelli 2018).

In occasione del primo sciopero dei rider, che chiedevano il ripristino di un pagamento minimo orario e una maggiore tutela del lavoro, il CEO di Foodora Italia risponde che i rider non sono lavoratori, ma persone a cui piace andare in bici e che ora possono farlo guadagnando qualcosa (Aloisi e De Stefano 2017). È una narrazione che si conforma pienamente al concetto di lavoretto, praticato da studenti, o da persone che vi ricorrono in forma accessoria, non fondandovi la propria sussistenza. Se di lavoretti si tratta, e non di lavoro, è normale che non vi siano le tutele tipiche del lavoro.

In realtà, i dati ci dicono che solo una quota marginale dei rider - così come di coloro che svolgono attività, o task, nell'ambito della Crowd Economy o della On Demand Economy, fa in modo accessorio: la maggioranza trae dalla propria attività mediata dalle piattaforme la principale o unica fonte di reddito (Schor 2018, Berg, De Stefano 2018) e molti rider, al pari dei clickworker di Mechanical Turk o CrowdFlower, lavorano attraverso le piattaforme sette giorni alla settimana, oppure compongono il loro reddito integrando "vecchie" e "nuove" forme di lavoro precario, che spaziano dall'attività di rider alla realizzazione di task nelle piattaforme, allo svolgimento di mansioni di cameriere o barista (Huws et al. 2018). E la maggioranza di loro dichiara di non essere nelle condizioni di riceverne un reddito dignitoso, e per questo sono alla continua ricerca di nuove mansioni da svolgere (Berg 2016). Allo stesso tempo, è interessante rilevare che, in tutti gli Stati europei, all'incirca la metà dei lavoratori impiegati in questo tipo di "lavoretti" si autodefinisce come un lavoratore impiegato a tempo pieno. In Italia, in cui pure il dato è più basso che in altri paesi, si autodefinisce così il 41\% dei crowd-workers, mentre solo il $7 \%$ si autodefinisce come un lavoratore autonomo (Huws at al. 2018).

Negli ormai numerosi pronunciamenti in materia da parte di giudici, se in Italia si è dichiarata l'inesistenza dei requisiti tali da considerare i rider lavoratori dipendenti, nel Regno Unito ha avuto una grande rilevanza la sentenza con cui la magistratura ha dichiarato "lievemente ridicolo" l'idea che si possa considerare "Uber a Londra come un mosaico di 30.000 piccoli imprenditori legati da una piattaforma comune" (Berg, De Stefano 2018).

Nel complesso, il fronte del conflitto che vede opporsi i lavoratori delle piattaforme alle multinazionali che le gestiscono è in piano sviluppo, e prende forma sul piano sindacale, sul piano giudiziario e attraverso lo sviluppo di movimenti e mobilitazioni dal basso e di pratiche di mutualismo e di autoorganizzazione (Aloisi, De Stefano 2017; Zamponi 2018). 


\section{CONCLUSIONI. LUCI E (MOLTE) OMBRE}

Un articolo pubblicato di recente su The Independent (Das 2017) ben sintetizza i rischi connessi alle trasformazioni del lavoro nell'ambito dell'economia delle piattaforme, che trovano espressione nello spettro di un "mondo dickensiano per i lavoratori". L'economia della condivisione, concetto con cui, spesso in modo improprio, ci si riferisce alle nuove forme del capitalismo digitale, ricorda l'autore, è comunemente visto più nei termini di un movimento sociale che come un modello economico. Un movimento sociale che trasforma le relazioni tra individui, genera reti fiduciarie e mobilita la solidarietà, ponendo al centro la persona e la comunità; un movimento animato da individui che perseguono l'incontro, i principi del bene comune, della sostenibilità, della reciprocità, dell'equità e della solidarietà, e non l'interesse economico immediato.

Si tratta, come abbiamo visto, di una realtà che è effettivamente in via di sviluppo, che si connette alle pratiche di consumerismo politico, alle esperienze di imprenditoria sociale, ai movimenti per i beni comuni e per la sostenibilità, dando forma a un vasto, multiforme, variegato mondo di economia solidaria, oltre che all'affermazione di un nuovo modo di pensare e di gestire il fare impresa (es. impresa 4.0).

Ma questo tipo di pratiche, sul piano dell'impatto effettivo, risultano minoritarie, rispetto alla forma prevalente assunta oggi dall'economia delle piattaforme, che si conforma, molto spesso, al modello della GIG Economy: in questa l'utilizzo delle tecnologie digitali più che al servizio di un cambio di paradigma, orientato al superamento dell'approccio economista ed utilitarista, è funzionale al perfezionamento di questo. Non aumenta i margini di flessibilità, di autonomia e di creatività del lavoratore. Ne rafforza, piuttosto, la dipendenza, il controllo e lo sfruttamento riducendone al contempo reddito, tutele, sicurezza. L'economia delle piattaforme, infatti, riuscendo a porre in diretta connessione il prestatore d'opera con chi ne fa richiesta, riduce ogni margine improduttivo, perfeziona il pagamento a cottimo, sovente aggirando la normativa posta a tutela dei lavoratori, non riconoscendo alcun rapporto di dipendenza. Ciò, peraltro, portando a compimento una pregressa concorrenza al ribasso nel mercato del lavoro, permettendo di superare l'ultimo ostacolo posto al suo pieno compimento: quello infrastrutturale e logistico.

Sul piano del consumo, le motivazioni prevalenti per cui si fa ricorso all'economia delle piattaforme sono di natura strettamente economica (cfr. Alaminos, Penalva 2018). "Gli scambi sono economici. I compratori sono interessati prevalentemente all'accesso a servizi a basso costo piuttosto che a obiettivi sociali. I fornitori sono motivati dal denaro, utilizzando le loro risorse e il loro lavoro per inserirsi in un ambiente economico spietato e povero" (Das 2017). Lo sviluppo di questo nuovo tipo di capitalismo, con le conseguenze 
potenzialmente distruttive sul lavoro che comporta, trova spiegazione con la concomitanza della diffusione delle tecnologie che lo rendono possibile con l'esplosione della crisi economica. Questa, come è stato evidenziato nei paragrafi precedenti, soprattutto nelle giovani generazioni, ha alimentato un radicale cambiamento di prospettiva nel rapporto con il consumo e con il lavoro. Ha favorito il superamento dei modelli tradizionali di consumo e al contempo delle rappresentazioni del lavoro caratterizzanti le generazioni precedenti, e tale superamento ha al contempo stimolato la sperimentazione di pratiche di innovazione economica e sociale, e l'accettazione di lavori sottoretribuiti e sottotutelati, come molti di quelli oggi offerti dalle piattaforme.

Gli stessi individui che lavorano nell'economia delle piattaforme, d'altra parte, ne sono utenti. La crisi economica ha promosso una trasformazione strutturale, riducendo la qualità del lavoro e ampliando per questa via la disponibilità di prodotti e, soprattutto, servizi a basso costo, alimentando un circolo vizioso, in cui la crisi economica, assumendo tratti strutturali, alimenta la disponibilità ad accettare un peggioramento delle condizioni e delle prospettive lavorative, all'interno di un sistema che si orienta sempre più, anche nel consumo, verso prodotti e servizi low cost, prodotto con impieghi low cost.

Economia della condivisione e della collaborazione, impresa sociale, diffusione del paradigma del societing, co-working, non rappresentano, in sé, forme di civilizzazione dell'economia, ma un cambio di paradigma, che contiene rischi e opportunità. Spesso si guarda a queste pratiche come esempi di resilienza, come alla capacità, cioè, di modellarsi e di adattarsi al mutamento, e che non oppongono un modello alternativo a quello dominante, ma vi offrono una risposta adattiva: è il caso, per esempio, del co-working, che, come, in generale, la sharing economy, è espressione di una "solidarietà debole": "il co-working non si costituisce mai come un'alternativa al mercato. Anzi, nei co-working si impara principalmente come posizionarsi in modo efficiente sul mercato. Anche se i coworker riconoscono di condividere una situazione comune, ossia di essere precari e generalmente sottopagati, questa consapevolezza non si traduce mai in una critica al mercato: né al mercato come istituzione - perché le responsabilità sono dovute principalmente ai "burocrati" o ai "politici" - né al prezzo di mercato: non esiste, come nel movimento operaio di fine Ottocento, una concezione del giusto valore del lavoro che può servire come base per una critica del suo prezzo di mercato. La "solidarietà debole" "è una solidarietà di mercato, una solidarietà imprenditoriale" (Arvidsson 2015) che assume la centralità della dimensione della relazionalità e dei meccanismi reputazionali, che può nascondere forme di sfruttamento, o elementi di biopolitica, ovvero di sussunzione della mente e del corpo del soggetto, nel suo sforzo di messa a valore di tutti gli aspetti che definiscono il suo sé e la sua relazionati. In tal modo, aggiunge Arvidsson, "la collaborazione non è fuori ma dentro al mercato, la capacità di collaborare diventa 
misurabile come un prezioso bene reputazionale e, per questo, viene gestita in modo riflessivo. In più, la reputazione non è dovuta unicamente alla capacità di condividere capitali sociali e culturali ma anche, e forse principalmente, alla capacità di creare un'esperienza collaborativa. Con la "creatività" del co-working, i co-worker intendono principalmente l'ambiente positivo ed energizzante che rende possibile autoconvincersi, nonostante le difficoltà economiche, che la propria attività e la propria scelta abbiano un senso. Si collabora anche condividendo le proprie competenze e skills, ma la collaborazione consiste soprattutto nella creazione di un'esperienza positiva quotidiana. L'obbligo di creare affettività positiva è ben sedimentato nell'habitus dei co-worker stessi: bisogna comportarsi in modo particolare, gestire le emozioni (non va bene mostrarsi eccessivamente depresso a causa, ad esempio, dell'assenza di lavoro), occorre parlare di precisi argomenti (le questioni di lavoro bene; quelle personali, oltre un certo limite, meno bene). Soprattutto, non bisogna creare energia negativa". D'altra parte. queste riflessioni rimandano all'interiorizzazione del nuovo spirito del capitalismo, descritto da Boltanski e Chiapello (2014), che spinge l'individuo a essere autonomo, ma al tempo stesso connesso, flessibile, adattabile: ad attivare relazioni e a collaborare, ma in una prospettiva che non pone la relazionati e la socialità come fini, ma come strumenti al servizio della competizione individuale.

Economia della condivisione, sviluppo di pratiche ibride, centralità della connessione pubblica e della relazionalità, affermazione del principio del societing, in definitiva, possono fondare, dietro la retorica della disintermediazione, della solidarietà, della valorizzazione dello scambio e dell'attivazione, nuove forme di assoggettamento, di sfruttamento e di autosfruttamento, che giungono fino ad istituzionalizzare il lavoro gratuito. Questo viene prodotto dai prosumers, nelle loro interazioni quotidiane, e in forma più consapevole per costruirsi un profilo di impiegabilità. Il lavoro gratuito rischia di diventare un vero e proprio elemento costitutivo della società tardo-moderna (Chicchi e Simone 2015; Coin 2016; Fumagalli 2017). La stessa porosità del confine tra agire economico e agire sociale, tra lavoro e vita personale, che nella faccia illuminata della luna avviene in direzione di una "civilizzazione" di un agire economico che si fa anche sociale, nel lato oscuro della luna rischia vieppiù di tradursi in pratiche che estendono il principio della messa a valore all'intera vita quotidiana, portando agli estremi i rischi di mercificazione, alienazione e sfruttamento degli individui, estendendoli a ogni ambito dell'agire sociale.

L'effetto finale rischia di essere quello di una crescente polarizzazione, tra una minoranza di individui che vivono il lato illuminato della luna e una quota crescente di individui che non riescono ad emergere dal lato oscuro, e per i quali il riferimento a concetti come economia della condivisione o economia della collaborazione rischia di ridursi a una retorica, a un eufemismo, che contribuisce 
a legittimare un reale peggioramento delle condizioni di lavoro, circondandolo di concetti suadenti e innovativi (Sundararajan 2016; Stewart, Standon 2017). Un esempio emblematico è la ridenominazione dei tradizionali facchini, che facevano le consegne in bicicletta segnandosi su un foglio gli indirizzi, in riders, che seguono le indicazioni ricevute via App.

La soluzione per uscire da questo rischio non può essere lasciata alla capacità di autoregolazione delle piattaforme, in un'ottica di deregolamentazione, ma va cercata in un ripensamento degli strumenti di protezione sociale e di regolazione del lavoro e, più in generale, nella capacità di regolare fenomeni il cui impatto investe una pluralità di sfere e di ambiti. Si tratta, in sintesi, di affiancare i processi di de-istituzionalizzazione in atto con nuove forme di istituzionalizzazione, che sappiano fornire un inquadramento delle nuove pratiche sorte come esito dei processi di innovazione tecnologica, economia e sociale, promuovendone gli aspetti virtuosi e neutralizzandone gli effetti negativi, che appaiono evidenti soprattutto in termini di sotto-retribuzione, di assenza di protezione, di spinta alla mercificazione e di sviluppo di forme di controllo pervasivo sul lavoratore (Berg, De Stefano 2018). Un necessario punto di partenza, a tal fine, è l'assunzione di consapevolezza che le tradizionali distinzioni tra lavoratore dipendente e lavoratore autonomo, così come quelle tra occupato e disoccupato, sono saltate, generando una serie di posizioni intermedie, profondamente ambigue. Ciò significa che gli strumenti di welfare, concepiti e strutturati per tutelare i lavoratori, da una parte, e per offrire strumenti di inserimento lavorativo per i disoccupati, non sono più adatti a rispondere ai bisogni di un numero crescente, nei giovani maggioritario, di lavoratori che si trovano in una terra di mezzo, che li vede lavorare sette giorni su sette, spesso per la stessa impresa, ma che, di fatto, al termine di ogni consegna, o task, risultano non impiegati (Huws et al. 2018).

Un necessario punto di partenza, per riflettere sul quadro regolativo, è approdare ad una chiarificazione concettuale, capace di discriminare tra fenomeni, pratiche ed esperienze assai diverse, che oggi continuano a essere erroneamente riunite sotto la stessa etichetta, anche nei documenti della Commissione Europea, che nelle sue ricerche ha adottato una definizione di "Cooperative Digital Economy" estesa, ricomprendendovi anche le pratiche for profit (Alaminos, Penalva 2018). In questa prospettiva, l'approfondimento che si è realizzato converge nella proposta di tipologizzazione proposta da Bouwen e Lievens (2013). Questi suggeriscono di suddividere il vasto e, altrimenti indifferenziato, mondo dell'economia digitale, sulla base di due assi: decentrato/locale vs. centralizzato/globale e capital oriented/for profit vs. social oriented/for benefit. 
Ne risultano quattro tipi:

1) "Netarchical capitalism" (centralizzazione/globalità e orientamento for profit): un capitalismo digitale che coniuga utilizzo della rete e verticalità. Qui le piattaforme digitali sono utilizzate da imprese private a scopo di profitto. È il caso di Facebook, AirBnB, TaskRabbit, ecc;

2) "Dinstributed capitalism" (decentralizzazione/localismo e orientamento for profit): vi rientrano esperienze come le monete alternative (Bitcoin);

3) "Global commons" (centralizzazione/globalità e orientamento ai common). Vi rientrano esperienze come Wikipedia, Creative Commons, i Transition Network;

4) "Local Resilience" (decentralizzazione/localismo e orientamento ai common). $\mathrm{Vi}$ rientrano tutte le pratiche di resilienza e di economia alternativa sperimentate a livello locale (Transition Towns, banche del tempo, ecc).

Molte delle distorsioni connesse all'affermazione dell'economia delle piattaforme trovano spiegazione nel ritardo, probabilmente fisiologico, nell'adattarsi a processi trasformativi che pongono di fronte ad assetti e pratiche che avvengono, spesso, in un vuoto normativo, e che, in alcuni casi, generano inedite linee di conflitto, sia all'interno delle imprese, sia nei territori. Ci riferiamo alle vertenze che, in Italia e in altri paesi europei, hanno visto i riders mobilitarsi per vedersi riconosciuto il loro status di lavoratori, e non di prestatori d'opera, ai conflitti che vedono opporsi Uber e i tassisti, ma anche alle mobilitazioni dei cittadini che, in alcune città, reclamano il loro "diritto alla città", a fronte del processo di espulsione dei cittadini favorito dall'espansione del fenomeno AirBnB.

In merito alle principali direttrici di intervento sul piano della regolazione e della tutela dei lavoratori, su cui è intervenuto anche il Parlamento Europeo (2017), una lista esaustiva è proposta da Stewart e Standon (2017), che indicano l'opportunità di espandere il raggio di azione delle leggi esistenti in materia di lavoro, facendovi rientrare un insieme di fattispecie lavorative che ne sono oggi di fatto escluse. Al centro di questa strategia vi è la necessità di ridefinire la condizione di lavoratore dipendente, applicandola a una serie di realtà in cui i lavoratori operano, formalmente, da freelance. La stessa definizione di impiego risulta da rivedere, al fine di includervi i lavoratori attivi nel crowdwork e nell'ondemand economy o identificando una nuova categoria di lavoratori indipendenti, che raccolga e regoli i lavoratori attivi in mansioni lavorative organizzate tramite le piattaforme digitali. infine, una strada può essere il ripensamento complessivo della categoria di lavoratore dipendente, ponendo al centro del sistema di tutele i lavoratori nella loro generalità, superando in questo modo una distinzione tra 
lavoratori dipendenti e lavoratori autonomi, che è di fatto oggi incapace di includere i lavoratori dell'economia delle piattaforme.

I tratti caratteristici della GIG Economy sono in sintesi, la frammentazione del lavoro in micro-lavori, o lavoretti, l'individualizzazione - e quindi l'indebolimento della dimensione collettiva del lavoro e dei suoi conflitti - la precarizzazione e al contempo l'estensione del lavoro in luoghi e in orari che un tempo afferivano alla dimensione privata, ma anche l'estensione del principio della competizione, tra i lavoratori di ogni parte del mondo, ma anche come principio continuo che regola l'interazione tra i lavoratori e tra questi e il datore di lavoro - o più spesso la piattaforma stessa, con cui interagiscono direttamente i lavoratori, si pensi alle condizioni di lavoro nei magazzini di Amazon o, in generale, nella logistica e nelle consegne - (Webster 2016; Stewart, Standon 2017).

Più nel dettaglio, gli elementi essenziali sono:

1) Un'estrema forma di frammentazione e parcellizzazione, al punto che, non di rado, sia i crowdworkers sia i lavoratori dell'On-Demand Economy, non ricevono un salario ma sono formalmente autonomi. Sottoscrivono un contratto di prestazione di lavoro ti di tipo occasionale ogni volta che eseguono quello che viene definito un "task", un compito (un piccolo intervento di riparazione, una consegna, come nel caso dei riders di Foodora, Deliveroo, ecc), un compenso per ogni task assegnato mediante la piattaforma e portato a compimento (Cavallini 2017). Le tecnologie, per i lavoratori attivi nella logistica, nelle consegne a domicilio, o nella realizzazione di task per le piattaforme digitali, dal proprio computer, non solo si pongono a fondamento di nuove forme di sfruttamento e di alienazione, ma della stessa privazione dello status di lavoratore: utilizzando termini apparentemente più moderni e "smart", i lavoratori a cottimo della rete diventano "turker", e i fattorini "riders".

2) Una netta separazione tra progettazione ed esecuzione, che impedisce al singolo lavoratore di avere una minima conoscenza dell'intero processo. Si pensi ai "task" che non il lavoratore, ma il "turker", realizza attraverso piattaforme come Mechanical Turk di Amazon, senza sapere per chi e per quale scopo realizza un lavoro. Non solo il singolo lavoratore svolge un'attività lavorativa estremamente circoscritta, ma lo fa in condizioni di solitudine e di isolamento, senza entrare a contatto con altri lavoratori e al di fuori di un ambito propriamente lavorativo.

3) Una progressiva indistinzione tra tempo di lavoro e tempo di non lavoro, che si ha dal momento che si lavora da casa, con propri strumenti, e si è spinti a lavorare il più possibile, nei tempi dettati dalla piattaforma, non solo perché i redditi sono molto bassi e si è sostanzialmente esclusi da ogni forma di 
tutela sociale, ma perché si è spinti a essere disponibili, performanti, e a ottenere buone reputazioni, quando si lavora in realtà come Uber, per esempio. Del resto, si lavora a casa, con il proprio computer, il proprio smartphone, la propria bicicletta, la propria auto, ecc.

4) La sostanziale scomparsa del conflitto. Lavoratori che operano in forma precaria, parcellizata e frammentata, senza diretto contatto con datori di lavoro e colleghi, al di fuori di contratti di tipo subordinato e salariato, e fintamente autonomi, che interagiscono prevalentemente con app e algoritmi, non dispongono delle condizioni e degli strumenti che consentono loro di saldare la loro esperienza in forme di mobilitazione collettiva. I lavoratori sono isolati l'uno dall'altro e la frammentazione della forza lavoro depotenzia la forza negoziale, vista la difficoltà dei lavoratori di organizzarsi in forme sindacali. Anche se, a ben vedere, si osservano interessanti segnali di mobilitazione in nuovi contesti lavorativi, come lo sciopero dei lavoratori di Amazon, in occasione del Black Friday, o dei riders di Foodora.

5) Un generalizzato peggioramento delle condizioni di lavoro, in termini salariali, di tutela dei diritti, di conciliazione tra lavoro e tempo di vita. Molti dei lavoratori impiegati nel platform capitalism sono sottoretribuiti, lavorano in condizioni di insicurezza, non hanno alcuna prospettiva di carriera. Svolgono mansioni ripetitive, sottoqualificate e che non offrono alcuna possibilità di acquisizione di competenze. Non sono protetti in caso di malattia, non hanno alcuna copertura sociale e pensionistica.

6) Lo spostamento dei rischi e degli oneri relativi all'esercizio della professione dall'impresa al singolo lavoratore, che è responsabile della rispondenza agli standard imposti, delle eventuali negligenze o errori, che in caso di incidente o di malattia non è coperto da alcuna protezione, che deve per di più, in quanto psedoautonomo, procurarsi e mantenere gli strumenti che gli consentono di lavorare.

7) Un'ambivalenza costitutiva tra dimensione della cooperazione e della condivisione e tendenza all'isolamento e all'individualizzazione. Il capitalismo digitale, infatti, enfatizza, celebra, esalta il principio della collaborazione. A ben vedere, però, tale retorica si pone per lo più a fondamento della creazione e dell'appropriazione privata di profitto (si pensi al lavoro dei prosumers), e, sul piano degli approcci al lavoro, diventa paradossalmente, uno strumento di competizione, a livello individuale e di impresa: le soggettività e la cooperazione spontanea sono da un lato incentivati a svilupparsi, dall'altro continuamente ricondotti alla creazione di valore di scambio (Caruso e Giorgi 2013, cfr. Fumagalli, Morini, 2009), al punto che in esperienze come il co-working si giunge a trasformare il bisogno di socialità in un prodotto da acquistare. 


\section{BIBLIOGRAFIA}

Acquaro D., Dell'Oste C. (2017), "Dalla casa all'auto, tutte le novità della sharing economy". Il sole 24 ore, consultabile in http://www.ilsole24ore.com/art/notizie/2017-06-26/stretta-sharingeconomy-122838.shtml?uuid=AEXpeCmB

Alaminos A., Penalva C. (2018), "Riflessioni sull'economia collaborativa come strumento di resilienza generazionale. Un contributo alla sua conoscenza e al suo utilizzo in Italia e in Spagna". in Pirni A., Raffini L. (a cura di), I giovani e la reinvenzione del sociale, in corso di pubblicazione.

Aloisi A. (2016), "Commoditized Workers. Case Study Research on Labour Law Issues Arising from a Set of 'On-Demand/Gig Economy' Platforms", Comparative Labor LawE Policy Journal, 37(3).

Aloisi, A., V. De Stefano and S. Silberman (2017), 'A Manifesto to Reform the Gig Economy', Pagina 99 , 29 May, http://www.pagina99.it/2017/05/29/amanifesto-to-reform-the-gig-economy/.

Alteri L., Cirulli A., Raffini L. (2018), "Adattamento, mitigazione o resistenza? La città neoliberista e i volti plurali delle pratiche di resilienza", paper presentato a Mobilità, migrazioni e territori, Convegno della sezione di sociologi urbane e del territorio della Associazione Italiana di Sociologia, Padova, 22-23 febbraio.

Armano, E.; Murgia, A. (2016) (a cura di), Le reti del lavoro gratuito. Spazi urbani e nuove soggettività, Verona: Ombre Corte.

Armano, E., Murgia, A., Teli, M. (2017). Platform capitalism e confini del lavoro negli spazi digitali. MIMESIS.

Arvidsson A.(2015), "La solidarietà debole", DoppioZero, consultabile in http://www.doppiozero.com/materiali/lavoro-freelance/la-solidarietadebole.

Arviddson A. e Giordano A. (2013), Societing Reloaded. Pubblici produttivi e innovazione sociale, Egea, Milano.

Bascetta M. (2015), L'economia politica della promessa, ManifestoLibri, Roma.

Beck U. (1999), La società del rischio, Carocci, Roma.

Berg, J. (2016), "Income Security in the On-Demand Economy: Findings and Policy Lessons from a Survey of Crowdworkers", Geneva: International

Labour Office, http://www.ilo.org/wcmsp5/groups/public/---ed_protect/--protrav/---travail/documents/publication/wcms_479693.pdf.

Berg J., De Stefano V. (2018), "Employment and Regulation for Clickworkers", in Neufeind M., O'Reilly J., Ranft F. (a cura di), Work in the Digital Age. Challenges of the Fourth Industrial Revolution, Rowman \& Littlefield, London-New York.

Bologna S. (1997), Il lavoro autonomo di seconda generazione. Scenari del postfordismo in Italia, Feltrinelli, Milano. 
Borzaga C. e Defourny J 2001 - (a cura di), L'impresa sociale in prospettiva europea. Diffusione, evoluzione, caratteristiche ed interpretazioni teoriche, Edizioni 31, Trento.

Borzaga-L. e Fazzi L. (2011), Le imprese sociali, Caress, Roma.

Borzaga C. e Zandonai F. (a cura di) 2009 - L'impresa sociale in Italia. Economia e istituzioni dei beni comuni, Rapporto Iris Network, Donzelli, Roma.

Boltansky L., Chiapello E. (2014), Il nuovo spirito del capitalismo, Mimesis, MilanoUdine.

Botsman, R. e Rogers, R., (2010), What's Mine Is Yours: The Rise of Collaborative Consumption, Harper Collins, New York.

Botsman R., 2013, The Sharing Economy Lacks a Shared Definition, Fast Company $\mathcal{E}$ Inc., disponibile all'indirizzo internet: www.fastcoexist.com/3022028/thesharing-economy-lacks-a-shared-definition.

Bouwen M., Lievens J. (2013), De wereld redden. Met peer-to-peer naar een postkapitalistische samenleving, Houtekiet.

Brenner N. (2004), New State Spaces: Urban Governance and the Rescaling of Statehood. Oxford

University Press, New York, NY.

Castel R. (2015), Incertezze crescenti. Lavoro, cittadinanza, individuo. Editrice Socialmente, Bologna.

Cavallini G. (2017), "Foodora, Deliveroo \& Co.: le fattispecie della gig-economy italiana, tra previsioni contrattuali ed effettive modalità di esecuzione del rapporto", paper presentato al convegno internazionale di studio Impresa, lavoro e non lavoro nell'economia digitale, Università degli Studi di Brescia, 12 e 13 ottobre.

CGIL Lombardia (2017), Industria 4.0. Scenari di competitività e di occupazione per le imprese del sistema industriale Filctem in Lombardia.

Chicchi F., Simone A. (2017), La società della prestazione, Ediesse, Roma.

Coin (2017), Salari rubati, Economia politica e conflitto ai tempi del lavoro gratuito, Ombre Corte, Verona.

Cuzzocrea V. (2015), "“Occupabili” più che occupati? Ambiguità di un concetto di policy nel caso italiano", Sociologia del lavoro, n. 138.

Dagnino, E. (2015), "Il lavoro nella on-demand economy: esigenze di tutela e prospettive regolatorie", Labour \& Law Issues, 1(1), 86-106.

Das S. (2017), "The sharing economy creates a Dickensian world for workers - it masks a dark problem in the labour market", The Independent, 12 February.

De Stefano V. (2015), "The Rise of the 'Just-in-Time Workforce': On-Demand Work, Crowd work and Labour Protection in the 'Gig-Economy'" (Conditions of Work and Employment Series 71). Geneva: International Labour Organisation. Available at: http://www.ilo.org/wcmsp5/ groups/public/-ed_protect/-protrav/travail/documents/publication/wcms_443267.pdf (accessed 3 July 2017). 
European Commission (2011), "Communication from the Commission to the European Parliament, the Council, the European Economic and Social Commitee and the Committee of the Regions, Social Business Initiative, Creating a favourable climate for social enterprises, key stakeholders in the social economy and innovation", Bruxelles.

European Parliament (2017), European social rights: workers' protection needs to be extended to new jobs. Press release, 19 January.

Fabris G. (2008), Societing. Il marketing nella società postmoderna, Egea, Milano.

Fana M. (2017), Non è lavoro, è sfruttamento, Laterza, Roma-Bari.

Farrell D and Greig F (2016), Paychecks, Paydays, and the Online Platform Economy: Big Data on Income Volatility, New York: JPMorgan Chase \& Co. Available at: https://www.jpmorganchase.com/corporate/institute/document/jpmcinstitute-volatility-2-report.pdf

Florida R, (2002), The Rise of Creative Class, Basic Book.

Fondazione Unipolis (2015), "Dalla Sharing Economy all'Economia Collaborativa l'impatto e le opportunità per il mondo cooperativo", I Quaderni di Unipolis (ottobre 2015), ricerca disponibile all'indirizzo: http://www.fondazioneunipolis.org/wpcontent/uploads/2015/12/Ricerca-Economiacollaborativa-eCooperazione.pdf, (04/07/2016).

Formenti C. (2011), Felici e sfruttati. Capitalismo digitale ed eclissi del lavoro, EGEA. Milano.

Fuchs C. (2010), “Web 2.0, Prosumption, and Surveillance”, Surveillance E Society 8(3), articolo disponibile all'indirizzo: http://ojs.library.queensu.ca/index.php/surveillance-andsociety/article/view/4165/41

Fumagalli A. (2017), Economia politica del comune. Sfruttamento e sussunzione nel capitalismo bio-cognitivo, DeriveApprodi, Roma.

Fumagalli A., Morini C. (2009), "La vita messa al lavoro: verso una teoria del valorevita.

Il caso del valore affetto", in Sociologia del lavoro, n. 115, pp. 94-115.

Huws U., Spencer N.H., Syrdal D.S. (2018), Working in the gig economy: Insights from Europe, in Neufeind M., O'Reilly J., Ranft F. (a cura di), Work in the Digital Age. Challenges of the Fourth Industrial Revolution, Rowman \& Littlefield, London-New York.

Iaione C. (2016), "Le politiche pubbliche al tempo della sharing economy. Nell'età della condivisione il paradigma del cambiamento è la collaborazione", in E. Polizzi, M. Bassoli, Le politiche della condivisione. La sharing economy incontra il pubblico, 2016, p. 33;

Inglehart R. (1998), La società postmoderna, Editori Riuniti, Roma.

Joseph, J. (2013), "Resilience as embedded neoliberalism: A governmentally approach", Resilience, 1, 38-52. 
Kostakis V. e Bauwens M., 2014, Network Society and Future Scenarios for a Collaborative Economy, Palgrave Macmillan, Houndmills, Basingtoke.

Le Gales P. (2016), "Neoliberalism and Urban Change: Stretching a Good Idea Too Far?", Territory, Politics, Governance, 4(2), 154-172.

Morozov M. (2016), Silicon Valley: i signori del silicio, Codice Edizioni, Torino.

Murray R. (2012), The Open Book of Social Innovation, The Young Foundation, Nesta.

Neufeind M., O'Reilly J., Ranft F. (a cura di, 2018), Work in the Digital Age. Challenges of the Fourth Industrial Revolution, Rowman \& Littlefield, London-New York.

O'Reilly J., Ranft F., Neufeind M., (2018), Introduction. Identifying the challenges for work in the digital age, in Neufeind M., O'Reilly J., Ranft F. (a cura di), Work in the Digital Age. Challenges of the Fourth Industrial Revolution, Rowman \& Littlefield, London-New York.

Pais I., Mainieri M. (2015), "Il fenomeno della sharing economy in Italia e nel mondo", Equilibri, 1, pp.11-20.

Pais I., Provasi G. (2015), "Sharing Economy: A Step towards the ReEmbeddedness of the Economy?", in Stato e Mercato, 2015, 105, p. 347;

Parrino L. (2015), "Coworking e lavoro indipentente", Fondazione Feltrinelli.

Passoni A. (2016), "Economia delle piattaforme e architettura digitale delle scelte. Appunti sull'alternativa cooperativa", Archivio Giuliano Marini (Unpublished).

Polany K. (2000), La grande trasformazione, Einaudi, Torino.

Raffini L. (2014), "La politica online alla prova della democrazia", in Alteri L., Raffini L. (a cura di), La nuova politica. Mobilitazioni, movimenti e conflitti in Italia, Edises, Napoli.

Raffini L. (2016), "L'economia della condivisione tra retoriche, ambiguità e lati oscuri", La Rivista delle Politiche Sociali, 1.

Rainie L., Welmann B. (2013), Networked: The New Social Operating System, The MIT Press, Cambridge.

Regini M. (2012), "Tre fasi, due modelli e una crisi generalizzata", Stato e mercato, 1, pp. 77-90.

Regione Toscana (2017), CollaboraToscana. Per un'agenda regionale sull'economia collaborativa, Libro verde.

Ricolfi L., Sciolla L. (1980), Senza padri né maestri. Inchiesta sugli orientamenti politici e sociali degli studenti, Bari, De Donato.

Rifkin J. (2001), L'era dell'accesso. La rivoluzione della new economy, Mondadori, Milano.

Rosa H. (2015), Accelerazione e alienazione. Per una teoria critica del tempo nella tarda modernità, Einaudi, Torino.

Schor J. e Fitzmaurice C.J., (2014), "Collaborating and Connecting: The Emergence of the Sharing Economy", in Reisch L.A. e Thøgersen J. (a cura 
di), Handbook of Research on Sustainable Consumption, Edward Elgar, Cheltenham, pp. 410-425.

Schor J. (2018), "The platform economy: Consequences for labour, inequality and the environment", in Neufeind M., O'Reilly J., Ranft F. (a cura di), Work in the Digital Age. Challenges of the Fourth Industrial Revolution, Rowman \& Littlefield, London-New York.

Schwab, K. (2018), Shaping the Fourth Industrial Revolution, Geneva, World Economic Forum.

Srnicek N. (2017), Platform Capitalism, Polity Press, Cambridge.

Staglianò R. (2016), Al posto tuo. Cosi web e robot ci stanno rubando il lavoro, Einaudi, Torino.

Standing (2011), Precariat. The new Dangerous Class, Bloomsbury Academic, London.

Sundararajan A. (2016), The Sharing Economy. The End of Employment and the Rise of Crowd-Based Capitalism, MIT Press, 2016.

Sunstein C. (2014), Semplice: L'arte del governo nel terzo millennio, Feltrinelli, Milano.

Vecchi B. (2017), Il capitalismo delle piattaforme, ManifestoLibri, Roma.

Vercellone C. (2006), Capitalismo cognitivo. Conoscenza e finanza nell'epoca postfordista, ManifestoLibri, Roma.

Zamagni S. (2013), Impresa responsabile e mercato civile, Il Mulino, Bologna.

Zamponi L. (2018), "Gig Economy Platforms and Transnational Labour Activism", in Pilot study: identifying and developing effective measures, Report of the TransSOL project: http://transsol.eu/files/2018/05/TransSOL-WP6Report.pdf

Zvolska L. (2015), "Sustainability Potentials of the Sharing Economy. The Case of Accommodation Sharing Platforms", IIEE Thesis, n. 31, http://lup.lub.lu.se/luur/download?func=downloadFile\&recordOId=8055 $286 \&$ fileOId $=8055287$.

Zysman J., Kenney M. (2016), "The Next Phase in the Digital Revolution: Platforms, Abundant Computing, Growth and Employment". ETLA Reports No 61.

Webster J. (2016), "Microworkers of the Gig Economy: Separate and Precarious", New Labor Forum, 25(3) 56-64

LUCA RAFFINI is Fellow Researcher at the University of Genova (Department of Political Sciences). He is member of the editorial board of the scientific journals «SocietàMutamentoPolitica. Rivista italiana di sociologia», and «Partecipazione e Conflitto». Among his field of interest there are the relations between youth and politics; the social and political usage of new media; participation and conflict; mobility and migration 